\title{
Die Französische Revolution als moralischer Schock
}

\author{
Zur politischen Dimension der Erforschung und Therapie des \\ menschlichen Geistes, ca. 1792-1806
}

\author{
Laurens Schlicht
} The French Revolution as Moral Shock. On the Political Dimension of the Research and Therapy of the Human
Mind, ca. 1792-1806

\begin{abstract}
The paper describes how, in the context of the development of the French human sciences (sciences de I'homme) around 1800, the reference to the Reign of Terror was constitutive for the formulation and legitimation of a procedure, which was based on what Jean-Étienne Esquirol called "moral shocks" (sécousse morale). The psychiatric and pedagogical discussion of non-physical effects on the spirit (esprit) of human subjects and patients essentially dealt with the question: could people have been liberated by the shocking surge of the Revolution from the demeaning and dependent habits of the Old Regime (ancien régime), or could this violent revolution have had a pathological effect? This article shows that, after 1800 , the latter interpretation became accepted. A professional self-image of psychiatric and pedagogical expertise formed in the relationship between physician and patient or teacher and student. This expertise justified employing shocks in professionally controlled settings, while the healing power of the revolutionary was negated. This article thereby distinguishes between four different perspectives on the pathological or healing effect of what was perceived as a "revolutionary shock": firstly, a positive perspective that interprets the shock of the Revolution as healthy, stimulating the vital forces of the people languishing in inactivity; secondly, a cautious perspective that emphasizes the necessity of curbing and controlling the passions of the people; thirdly, a perspective that recognizes in the outburst of passions an aberration from the natural state, and fourthly, a therapeutic perspective that recommends the use of passions by experts in very controlled spaces. Based on published texts and material from the Archives nationales as well as the Institut national de jeunes sourds, this article provides a political history of the development of moral shocks and argues that the development of epistemological and therapeutic technologies in the human sciences were essentially the result of a demarcation from revolutionary violence as well as the desire in the human sciences for a stable government.
\end{abstract}

Keywords: French Revolution, History of the Human Sciences, Psychiatry, Science and Politics, Violence

Im Jahr 1805 bezeichnete der Arzt Jean-Étienne Esquirol eine therapeutische Methode mit dem Begriff des "moralischen Schocks" (sécousse morale). In vorliegendem Aufsatz wird dargestellt, inwiefern im Rahmen der Entwicklung der französischen Humanwissenschaften (sciences de l'homme, science social) um 1800 der Bezug auf den terreur konstitutiv für die Formierung dieser Behandlungsmethode war. Die psychiatrische und pädagogische Diskussion über diese nicht physische Einwirkung auf den Geist (esprit) von menschlichen Forschungsobjekten und Patient_innen bezog sich dabei wesentlich auf die Frage, ob das Volk durch die schockartige Aufwallung der Revolution aus den unfreien Gewohnheiten des ancien régime befreit werden könne. Nach 1800, so wird im Folgenden gezeigt, bildete sich das professionelle Selbstverständnis der psychiatrischen und pädagogischen Expertise heraus, mit der Schocks in professionell kontrollierten Settings eingesetzt werden konnten, während die heilsame Wirkung des revolutionären Schocks negiert wurde. Im Aufsatz werden hierfür vier verschiedene Perspektiven auf die pathogenen oder heilsamen Wirkungen von Schocks unterschieden. Gestützt auf publizierte Texte und Material aus den Archives nationales sowie dem Institut national de jeunes sourds wird so im Aufsatz eine politische Geschichte der Entwicklung des moralischen Schocks entfaltet und die These vertreten, dass die Entwicklung von epistemischen und therapeutischen Techniken in den Humanwis- 
Im Jahr 1789 schrieb ein Augenzeuge der Französischen Revolution in schwärmerischem Ton: „Welch gewaltiges und mannigfaltiges Gemälde der verschiedenartigen Wirkungen der menschlichen Leidenschaften uns doch das Erwachen eines großen Volkes schafft, das sich aus einer langen Lethargie befreit! ${ }^{\prime 1}$ Es handelte sich um Philippe Pinel (1745-1826), den die Medizingeschichte als eine der wichtigsten Gründungsfiguren der französischen Psychiatrie sieht. ${ }^{2}$ Die Revolution habe nicht nur einen politischen, sondern einen körperlichen Zustand beendet, den Pinel in den schwärzesten Farben malt: „Der Körper verkümmerte in Untätigkeit durch die $\mathrm{Zu}$ nahme von Weichheit und Luxus und die unruhige Aktivität des menschlichen Geistes konnte nicht länger unterdrückt werden“. Die „gewaltigste Explosion des Patriotismus und des Mutes“ habe für den „beobachtenden Mediziner" die heilsamen Wirkungen der Revolution offenbart: „Es geht mir besser seit der Revolution" höre man nicht selten. ${ }^{3}$

In diesem Aufsatz wird dargestellt, wie in den Diskussionen der $\mathrm{Hu}-$ manwissenschaften seit 1795 der Bezug auf den terreur konstitutiv für die Entwicklung von Techniken zur Erforschung, Kontrolle und Therapie des menschlichen Geistes war. Ich werde den Begriff „Humanwissenschaften“ im Folgenden verwenden, um das heterogene Theorie- und Praxisfeld zu bezeichnen, in dem verschiedene Ansätze für eine empirische Wissenschaft vom Menschen diskutiert wurden, wie die science bzw. sciences de l'homme, die sciences morales oder die science sociale. Dabei möchte ich zeigen, dass der Bezug auf den Schock der Revolution wichtig für die Entstehung der sogenannten "moralischen Behandlung" war. Zum einen will ich darstellen, in welcher Weise therapeutische Interventionen, die auf dem Konzept der moralischen Behandlung fußten, um 1800 unter Bezugnahme auf Deutungen von Erschütterungen während der Französischen Revolution konzipiert wurden. Zweitens werde ich die These vertreten, dass der Schock als therapeutische Intervention eines medizinischen Experten nach 1800 im Rahmen eines reformierten Verständnisses medizinisch-psychiatrischer Expertise in kontrollierten Settings wieder möglich wurde, nachdem dieser zuvor als pathogen kritisiert worden war. ${ }^{4}$ Ich werde hierfür vier unterschiedliche Perspektiven auf die Wirkungen von Schocks vorstellen und zeigen, dass diese Perspektiven Reaktionen auf die jeweiligen revolutionären und post-revolutionären Ereignisse waren, die durchweg an der Frage entwickelt wurden, wie die Leidenschaften der französischen Bür- 
ger_innen genutzt, organisiert, kontrolliert und moderiert werden konnten. Den Begriff des „moralischen Schocks“ (sécousse morale) habe ich aus JeanÉtienne Esquirols (1772-1840) medizinischer Dissertation des Jahres 1805 entnommen (Des passions considérées comme causes, symptômes et moyens curatifs de l'aliénation mentale). Dieser Text wird im weiteren Verlauf noch näher dargestellt, seine Deutung des Schocks als plötzliche und häufig gewaltsame Veränderung und des "moralischen Schocks“ als einer solchen Veränderung, die durch eine moralische, das heißt nicht direkt physische Einwirkung zustande kommt, werde ich übernehmen. In Anlehnung an Esquirol werde ich also unter "Schock“ im Folgenden die Wahrnehmung einer meist gewaltsamen, plötzlichen Erschütterung verstehen und diesen Begriff wenn nötig qualifizieren, das heißt, wie Esquirol von „politischen“ oder „medizinischen“ Schocks sprechen. Da sich der Aufsatz auf wissenschaftliche Diskurs- und Praxisphänomene in einer vordisziplinären Phase bezieht, von denen einige nicht zur Disziplinbildung geführt haben, gibt es kein bereits klar definiertes Corpus, das eine Analyse der dargestellten humanwissenschaftlichen Ansätze erlauben würde. Ausgehend von der Classe des sciences morales et politiques des Institut national des sciences et des arts sowie von der Pariser Institution nationale des sourds-muets habe ich relevante Diskurse in Monographien und Periodika verfolgt sowie Archivbestände der Archives nationales (Paris) und des heutigen Institut national de jeunes sourds de Paris (früher Institution nationale/royale/impériale des sourds-muets) miteinbezogen.

Die Tatsache, dass eine Schule für Gehörlose ins Zentrum gerückt wird, verlangt eine kurze Begründung: Die Erziehung von sourds-muets war, wie etwa Chappey und Rosenfeld ausführlich darstellen, im Verlauf der Französischen Revolution deshalb ein besonderer Ort der Diskussion über Humanwissenschaften, weil zum einen die Pariser Institution die erste staatlich finanzierte Schule dieser Art war und zweitens führende Vertreter_innen der Humanwissenschaften (wie etwa Pinel, Cabanis, Sicard, Gérando, Staël) beispielsweise im Kontext der Société des observateurs de l'homme (1799-1804) und dem Institut national des sciences et des arts die Frage der universellen Erziehbarkeit und Therapierbarkeit des Menschen ins Zentrum stellten (Rosenfeld 2001; Chappey 2017). Drittens erschien diese Frage den Akteur_innen anhand des vermeintlichen „Naturmenschen", des sourd-muet, besonders gut exemplifizierbar, weshalb auch die öffentlichen Sitzungen der Institution nationale des sourds-muets Gelegenheiten boten, die allgemeine Frage nach der empirischen Erforschung des menschlichen Geistes zu diskutieren (Quartararo 2008). 


\section{Forschung zur Wirkung von Schocks auf den menschlichen Geist im Untersuchungszeitraum}

In der Wissenschaftsgeschichte der Humanwissenschaften existieren im Vergleich zur allgemeinen Rezeption des terreur nach 1795 nur wenige Beiträge über die Wahrnehmung des "Schocks“ der Französischen Revolution hinsichtlich der Entwicklung therapeutischer und epistemischer Techniken (Baczko 1994 [1989]; Chappey 2017). Da es sich hier zeitlich um die Phase der Disziplinbildung in den Humanwissenschaft handelt, ist die wissenschaftshistorische Erschließung zum einen auf die Vorgeschichte bestimmter Disziplinen und Institutionen fokussiert (vor allem die Psychiatrie), zum anderen gibt es Forschungen, die sich eher für den spezifischen zeitgenössischen Anspruch einer allgemeinen Wissenschaft vom Menschen interessieren (beispielsweise Chappey 2002). Aufgrund der zentralen Bedeutung der Veränderung von Subjektkonstruktionen im Bildungsprozess der Humanwissenschaften waren überdies Forschungen zur Geschichte des Subjekts zu beachten.

Als Vorläufergeschichte des Traumakonzepts analysiert Ronen Steinberg die medizinische und politische Deutung der Schockwirkung der Revolution (Steinberg 2015). Er vertritt hierbei die These, dass die Veränderungen, die durch das „Age of Revolutions“ herbeigeführt wurden, die Bedingungen für die Entstehung des Traumabegriffs bereiteten (ebd.: 30). Steinberg konzentriert seine Analyse auf die Veränderungen im Begriff des terreur, der seit den 1790er Jahren nicht nur eine starke Emotion, sondern auch ein Regierungssystem bezeichnete (ebd.: 32), und geht davon aus, dass eine positive Konnotation des terreur nach 1800 unter Napoleon nicht mehr möglich gewesen sei, nachdem dies zuvor, wie weiter unten dargestellt wird, durchaus möglich gewesen war. George Rosen hat die Genese der Deutung sozialer Umstände als Ursachen von Geisteskrankheit analysiert und geht im Kontext der Amerikanischen Revolution auf positive Deutungen des revolutionären Schocks ein (Rosen 1959).

Besser erforscht ist die Geschichte des Einsatzes von Leidenschaften im engeren Rahmen der Geschichte der Psychiatrie und der des Wahnsinns, insbesondere im Hinblick auf den zentralen Akteur Philippe Pinel. Die ausführlichste Darstellung der Behandlungsmethoden bei Pinel findet sich nach wie vor in Jan Goldsteins Studie Console and Classify von 1987, in der diese die Rezeption des aus England stammenden moral treatment in Frankreich untersucht. Die Medikalisierung der Leidenschaften (passions) war schon zuvor in einem Aufsatz von Jackie Pigeaud erläutert worden, die ihre Rolle bei der Heilung von Wahnsinn in den theoretischen Texten von Esquirol und Moreau de la Tours darstellt und die These vertritt, dass sich hier die Bedeutung des Einsatzes von Leidenschaften (zu denen auch 
Furcht zählt) vermindert (Pigeaud 1980). Beide Arbeiten stützen sich auf die empirischen und theoretischen Entwürfe Michel Foucaults, ohne dessen Einordnung des psychiatrischen Wissens dieser kritische Diskurs über Psychiatrie nicht möglich gewesen wäre. Dessen Thesen zur Änderung der episteme um 1795 (Foucault 1974 [1966]), der Änderung des ärztlichen Blickregimes (Foucault 1976 [1963]) und der Analysen in Wahnsinn und Gesellschaft (Foucault 1969 [1961]) bilden den Hintergrund, vor dem die Diskussion über die gesellschaftlichen und politischen Dimensionen des Wahns und der Erforschung des Menschen sich seit den 1960er Jahren entfaltet hat. Hierbei sind die Arbeiten Gladys Swains und Marcel Gauchets zentral, die Foucaults Analyse des Wahns insofern kritisch weiterentwickeln, als sie dessen These widersprechen, der Wahnsinn sei im "Zeitalter der Vernunft" zum Schweigen gebracht worden; Pinels Entdeckung sei es im Gegenteil gewesen, dass ein Dialog mit dem Wahnsinnigen möglich sei, womit er vom insensé zum aliéné geworden sei (Swain 1997; Gauchet 1997: 10). Für den deutschsprachigen Raum hat Doris Kaufmann eine wertvolle Arbeit zur Analyse der Psychiatrie im bürgerlichen Zeitalter vorgelegt (Kaufmann 1995).

Die Forschungen zur Rolle des Subjekts sind zu zahlreich, um sie hier zu referieren, im Wesentlichen werde ich mich jedoch auf Jan Goldsteins spätere Arbeit über das Post-Revolutionary Self stützen, die sich besonders intensiv mit der Entwicklung der Introspektion bei Victor Cousin (1792-1867) als neuartiger Innerlichkeitstechnik befasst (Goldstein 2005). Zum anderen beruht mein Argument auf der Forschung Jean-Luc Chappeys zum "sauvage de l'Aveyron“, der die Geschichte des in den Wäldern bei Aveyron aufgefundenen vermeintlich wilden Jungen als politische Geschichte erzählt. Chappey vertritt hier die These, dass identifikatorische Zuschreibungen wie "Idiot" oder „Wahnsinniger“ nach 1804 immer mehr im Rahmen eines sogenannten Fixismus vorgenommen wurden, das heißt, dass sie als festgelegt begriffen wurden, ohne Möglichkeit der Transgression (Chappey 2017: 220).

\section{Die utopisch-revolutionäre Deutung des Schocks der Revolution}

Pinel hatte mit seiner eingangs vorgestellten Beschreibung des Schocks der Französischen Revolution ein Narrativ aufgegriffen, das in der Folge medizinisch gedeutet das Verhältnis von revolutionärer Bewegung, Gewalt und Aufklärung verständlich machen sollte. Er ging von der Gültigkeit zweier Thesen aus: Erstens hätten alle Menschen das gleiche Potential, an einem aufgeklärten, rationalen Diskurs teilzunehmen, weil sie gleich geboren wür- 
den und nur die Umstände (circonstances) sie ungleich machten, sie besaßen - um das technische Vokabular der Zeit zu verwenden - die gleiche Perfektibilität (perfectibilité). ${ }^{5}$ Zweitens führte das Leben in despotischen Regimen, wie dem ancien régime oder in China, zur Unterdrückung von Verstandeskräften, zu artifiziell bedingter Ignoranz und damit zur Abhängigkeit von einer Elite. ${ }^{6}$ Über eine längere Zeit ausgedehnt, verhärtete sich diese Abhängigkeit tendenziell, bis nur noch ein Schock von außen diese Strukturen und die angenommenen Gewohnheiten (habitudes) aufbrechen konnte. ${ }^{7}$ Der im 18. Jahrhundert neu aufkommende „Mediziner-Philosoph“ (médecin-philosophe) erbot sich, medizinisches Wissen über die Funktionsweise des menschlichen Körpers auch für erkenntnistheoretische und politische Fragestellungen nutzbar zu machen (vgl. Gusdorf 1960: 113-128). Die Strategien der „Mediziner-Philosophen“ in den frühen 1790er Jahren, den Ausbruch der Revolution im Jahr 1789 fassbar zu machen, stützten sich häufig auf die vibrierende Kraft der Ratio, der Aufklärung oder einfach des Geistes (esprit), die untergründig stetig an die Oberfläche gedrängt hatte, um schließlich der verhassten Herrschaft der Despoten und Tyrannen ein Ende zu bereiten. Der Mediziner François Xavier Lanthenas (1754-1799) äußerte sich 1792 in diesem Sinne zum „Einfluss der Freiheit auf die Gesundheit, die Moral und das Glück“" (De l'influence de la liberté sur la santé, la morale et le bonheur), dass sich die Mediziner gut überlegen müssten, was sie tun wollten: palliativ die Folgen des Despotismus' zu mäßigen oder als wahre Philosophen die eigentliche Krankheitsursache zu bekämpfen (Lanthenas 1792: 71). Er war nämlich der Ansicht, dass nur „die wahre Freiheit, Aufklärung und die Philosophie“ (la vraie liberté, les lumières et la philosophie) zum moralischen und physischen Wohlbefinden führen könnten (ebd.: 74). Das Bild eines in moralischer und physischer Schwäche dahinschmachtenden Volkes, das nur durch einen gewaltigen Schock aus der Atonie seiner entwürdigenden Gewohnheiten (habitudes) gerissen werden konnte, übertrug sich bei ihm auch auf die Auffassung des despotischen Herrscherkörpers selbst, dessen „Mund unrein und dessen Atem übelriechend“ sei: „Niemals wäscht er seinen Körper, niemals lässt er ihn unmittelbar den heilsamen Eindruck der freien Luft genießen“ (ebd.: 75). ${ }^{8}$ Freiheit, Glück und Gesundheit waren in dieser Perspektive Teil ein und desselben Wechselwirkungsgefüges und die Französische Revolution war in der Tat, wie Nina Gelbart es beschreibt, ein "medizinisches Ereignis“ (Gelbart 1989). Auch der spätere Mitautor von Napoleons Staatsstreich Emmanuel-Joseph Sieyès (1748-1836) ging in seinem berühmten Was ist der Dritte Stand? (Qu'est-ce que le Tiers-État?) von der befreienden Kraft "guter Schocks“ (bonnes secousses, Sieyès 1789: 173) für das Volk aus; gegen die These, man müsse dem Volk die Wahrheiten der Aufklärung in kleinen Portionen servieren, um es nicht zu überfordern, wandte er ein, 
dass die irritierende, schockartige Kraft der Wahrheit die „gothischen“ Irrtümer der Vergangenheit zerstreuen könne (ebd.: 172-173). Man müsse den in den Vorurteilen des ancien régime befangenen kranken Körper des Volkes, den „boshaften Saft“ (humeur maligne) (ebd.: 179-180) neutralisieren, die Gesundheit und das Spiel der Organe wiederherstellen (ebd.: 180):

Ihr werdet nun sagen, die Geister wären noch nicht reif genug, um euch $\mathrm{zu}$ verstehen, ihr würdet viele Leute vor den Kopf stoßen [choquer]. Aber das muss so sein: Die Wahrheit, deren Veröffentlichung am meisten Nutzen bringt, ist doch nicht die, der man schon ziemlich nahe ist, und auch nicht die, die man gerade im Begriff ist, sich zu eigen zu machen. Vielmehr ist die Verbreitung einer Wahrheit umso notwendiger, je mehr Vorurteile und persönliche Interessen sie verletzt [irriter] (Sièyes 2010[1789]: 172). ${ }^{9}$

Belebung durch Irritation war so ein mit dem revolutionären Schock positiv verbundenes Motiv: Genauso meinte Pinel im oben zitierten Aufsatz, dass der Organismus durch die Revolution „wie durch eine elektrische Kraft" (comme par une vertu électrique) wiederbelebt worden sei. ${ }^{10}$ Ich möchte diese Perspektive utopisch-revolutionär nennen, weil sie sich der unmittelbaren Machbarkeit einer besseren Welt verschreibt, die nur durch eine Revolution verwirklicht werden kann.

\section{Kritik an der utopischen Gewalt der Revolution}

Nach dem terreur, das heißt nach dem Sturz und der Hinrichtung Robespierres am 28. Juli 1794, dem 10. Thermidor des Jahres II, war es für die nun regierenden Thermidorianer eine vordringliche Aufgabe zu erklären, wie die von Natur aus guten Leidenschaften des Volkes während des terreur derart hatten auf Abwege geraten können. ${ }^{11}$ Schockartige Erschütterungen aller Art und vor allem der revolutionäre Schock waren in ihren Augen verdächtig und von einer wissensbasierten Regierungstechnik einzudämmen.

Eines der wesentlichen Vokabulare, dessen sich dieser politische Diskurs bediente, war das philosophische Wörterbuch der „Ideologen“, das heißt jener Gruppe Wissenschaftler, die mit Bezug auf Étienne Bonnot de Condillac (1714-1780) grundsätzlich die These vertraten, dass jede Kenntnis, und in der starken Variante auch jedes geistige Vermögen, sich aus der Sinneswahrnehmung erklären ließe. In dieser Zeit lässt sich deshalb eine Konjunktur von sensualistischen Ansätzen beobachten, die erklärten, wie Menschen von externer Stimulation durch Sinnesreize beeinflusst würden oder aufgrund ihrer spezifischen „Sensibilität“ Sinnesreize auf bestimme 
Weise prozessierten. Diese Ansätze, die auch dazu beitrugen, eine science sociale zu begründen, setzten wesentlich auf die These, dass menschliches Verhalten aufgrund von Naturgesetzen oder zumindest gesetzesartiger Folgerungen vorhersehbar und kontrollierbar sei (vgl. Wokler 2000). Auch im politischen Diskurs, etwa über die Gestaltung öffentlicher Feste oder die Nomenklatur der Straßennamen, sah man die Abhängigkeit des Denkens als „transformierte Sinneswahrnehmung “12, bei der die Bürger_innen einer gezielten Steuerung potentiell gefährlicher Sinnesreize und ihrer zeichenhaften Repräsentation ausgesetzt waren. ${ }^{13}$

Akteure wie Jean Lambert Tallien und Paul de Barras (vgl. Baczko 1994 [1989]), die sich nach dem terreur als Retter der Republik darstellten, betonten die Gefahren einer unkontrollierten Steuerung von Menschen über Sinnesreize: eine pervertierte Form, Dinge wahrzunehmen, eine falsche Weise, diese Wahrnehmungen (sensations) zu Ideen $\mathrm{zu}$ verarbeiten und eine fehlerhafte Art, diese Ideen im Rahmen von Urteilen zu vergleichen. Da für die letzte Funktion nach Condillac die Verwendung von sprachlichen Zeichen zentral war, bestand eine Gefahrenquelle im täuschenden Gebrauch von Zeichen (Aarsleff 1982; Rosenfeld 2001). Der mögliche Missbrauch von Zeichen seitens der Regierenden wurde in der Debatte um den sogenannten abus des mots thematisiert, den Missbrauch von Wörtern vor allem des politischen Vokabulars (wenn etwa durch das Wort „Gerechtigkeit" Handlungen erleichtert wurden, die tatsächlich ungerecht waren, vgl. Rosenfeld 2001; Vecchio 1986: 381f.; David 1801) oder auch der Schrift. Ein Beispiel: Schrift als Transmissionsmedium von Sinnesdaten und zur Erklärung politischer Zustände wurde intensiv in Bezug auf China diskutiert. Eine Fraktion, zu der Antoine Destutt de Tracy (1754-1836) gehörte, meinte, dass die chinesischen "Hieroglyphen“ den "statarischen" - also sich nicht weiterentwickelnden - Charakter der chinesischen Nation (mit)bedinge (vgl. Osterhammel 1989: 30). Er ging davon aus, dass die durch den langen Despotismus in China verhärtete Ignoranz des Volkes zuletzt nur noch durch eine Invasion von außen aufgebrochen werden könnte; die nationalen Gewohnheiten hätten sich - anders als beispielsweise Marie Jean Antoine Nicolas Caritat, Marquis de Condorcet (1743-1794), Sieyès oder Pinel in den frühen 1790er Jahren geglaubt hatten - niemals von selbst in der Geschichte verändert, da dies zum einen einfach nicht belegt sei und zum anderen einem „ungestümen und vollständigen Umschwung“ zu viele Gewohnheiten entgegenstünden (Destutt de Tracy 1803: 305; dazu Messling 2013). ${ }^{14}$

Das Schema einer anthropologischen Erklärung von Fehlerquellen der Wahrnehmung und ihrer Verarbeitung strukturierte auch die Erläuterung des politischen Verhaltens von Menschen und Menschenmengen. Die zentrale Rede Jean-Lambert Talliens (1767-1820) über den terreur nach der 
Hinrichtung Robespierres im Jahr 1794 ist hierfür ein gutes Beispiel. Als einer der Architekten der politischen Ordnung im Anschluss an die Terrorherrschaft meinte Tallien, dass die republikanische Regierung ein System der ständigen Vigilanz benötige, in dem die Kräfte der öffentlichen Ordnung jede Anstrengung unternehmen müssten, um Aufruhr zu vermeiden. Denn in einer Republik seien „sozusagen alle Köpfe mit Pulver angefüllt, und die kleinsten Funken, die die Diskussionen der Convention nach links oder rechts schleudern, werden unweigerlich Feuer in irgendeinem Teil der Republik entfachen" (Tallien 1794: 612). ${ }^{15}$ Nachdem er während des terreur zunächst selbst eine politische Karriere begonnen hatte, war er inzwischen davon überzeugt, dass es die höchste Pflicht der Regierenden sei, die Entstehung solcher Flächenbrände zu verhindern. So wurde nach dem terreur, wie auch Sophie Wahnich gezeigt hat, die Vorstellung der souveränen und naturwüchsigen Macht des Volkes, sich selbst von seinen Ketten zu befreien, durch eine argwöhnische und paternalistische Perspektive auf eine Masse von Menschen ersetzt, die sich als korrumpierbar, iraszibel und manipulierbar erwiesen hatte (Wahnich 2009; Baczko 1994 [1989]: 54-60). Für Tallien war der terreur dabei eine pathogene Kraft, die zur „Störung aller Ideen und zur Umkehrung aller Gefühle (affections)“ führe, ein „äußeres Beben, das die verborgensten Fasern affiziert, das den Menschen erniedrigt und ihn dem Tier gleichsetzt", ein "Schock aller physischen Kräfte, die Erschütterung aller moralischen Vermögen“ (Tallien 1794: 613). ${ }^{16}$ Die Thermidorianer haben, wie Baczko, Wahnich und andere dargestellt haben, sich dabei bemüht, die Frage der Schuld an der Terrorherrschaft so $\mathrm{zu}$ rahmen, dass es möglich wurde, den Großteil des Volkes (und auch einige ehemalige Terroristen) von ihr freizusprechen (Baczko 1994 [1989]: 54-60; Wahnich 2009: 64-73). So wurde die rhetorische Konstruktion des „verführten“ Volkes verwendet, um eine kleine Zahl von Schuldigen einer großen Zahl von Verführten entgegenzustellen. Diese Haltung begründete Bertrand Barère (1755-1841) schon gleich zu Beginn der Thermidorianerherrschaft, indem er betonte, das Volk sei vom Triumvirat des terreur „getäuscht“ worden (le peuple était trompé) (Barère 1794: 369). Dies bedeutete andererseits in der Konsequenz, dass sich das Volk eben auch täuschen und zu grausamen Handlungen hinreißen ließ, ohne sich selbst dagegen schützen zu wissen. Eine Eingabe des Volkes von Tours an den Nationalkonvent vom 3. August 1794 schloss sich dieser Narration an, die das Triumvirat des terreur als "Monster" (monstres) oder „Tyrannen“ (tyrans) bezeichnete und den Missbrauch revolutionärer Sprache als Vehikel der Täuschung und Unterdrückung des Volkes brandmarkte: „Der arglistig unter der Maske von Tugenden und Heuchelei verborgene Despotismus wird sich nicht länger durchsetzen .... Diese Monster! .... sie haben eure 
Sprache nur verwendet, um uns besser zu unterdrücken!“ (Peuple de Tours 1794: 375$) \cdot{ }^{17}$

\section{Positive Einschätzungen des revolutionären Schocks, 1795-1799}

Talliens starke Ablehnung des terreur sowohl als Regierungssystem wie auch als Leidenschaft (passion) war eine der typischen Positionen innerhalb des Diskurses über die Historizität der Revolution und die medizinische Rolle der Leidenschaften. ${ }^{18}$ Im Rahmen der Französischen Revolution wurde diese medizinische Deutung der Furcht, der Angst, des Terrors usw. als Lesart des Regierungssystems terreur übernommen und die gesamte Geschichte der Revolution zu einer medizinischen Fallgeschichte mit ihrer notwendigen Krisis. Die utopisch-revolutionäre Perspektive Pinels war dabei auch nach dem terreur noch eine Option: Hierbei ist das Beispiel des Arztes Marc-Antoine Petit (1766-1811) erhellend, der während des Lyoner Aufstands gegen den Nationalkonvent im Jahr 1793 Chefchirurg des dortigen Krankenhauses Hôtel-Dieu war. Anstatt sich über das Bombardement zu beklagen, während dessen das Krankenhaus mutmaßlich von 42 Geschossen getroffen wurde, hob Petit in der Eröffnungsrede des Anatomiekurses im Jahr 1796 mit dem Thema „Über den Einfluss der Französischen Revolution auf die öffentliche Gesundheit" (Sur l'influence de la Révolution française sur la santé publique) eher die positiven Wirkungen von Furcht und Schrecken hervor: „Für den politischen Körper, den Revolutionen erschüttern, sind diese nichts anderes als was für den geschwächten menschlichen Körper die Medikamente sind, welche die Harmonie wiederherstellen sollen. Im einen wie im anderen Fall ist die erste Wirkung eine Unordnung, die erste Empfindung ein Schmerz" (Petit 1806 [1797]: 116). ${ }^{19}$

Petit meinte wie Pinel, dass dieser "Schock aller Leidenschaften“ offenbart habe, dass die Seele bislang ungeahnte Kräfte besitze (ebd.: 122) und die Revolution insbesondere die schädlichen Gewohnheiten eines versklavten Volkes in einer schockartigen Bewegung aufbreche (ebd.: 123). Entsprechend des medizinischen Sensibilitätsdenkens und des Vitalismus schienen ihm hierfür insbesondere atonische, tendenziell phlegmatische Personen empfänglich, was in der sexistischen Perspektive der damaligen Medizin bedeutete, dass gerade Frauen durch das Bombardement besonders affiziert worden seien (ebd.: 124).

Die historische Konstruktion von Texten, die den terreur willkommen hießen, war also grundlegend: In moralischer und physischer Hinsicht absolut schädliche Gewohnheiten des individuellen Körpers wie auch des 
ganzen Volkes, verstanden als Körper, mussten durch einen großen Schock überwunden werden. Adrien Lezay-Marnésia (1769-1814) - zu dieser Zeit im Journal de Paris publizistisch aktiv und in den humanwissenschaftlichen Zirkeln vernetzt - veröffentlichte im Jahr 1797 ein Buch mit dem Titel Über die Ursachen der Revolution und ihre Folgen (Des causes de la Révolution et de ses résultats), in dem er der Notwendigkeit revolutionärer Gewalt ein historiographisches Gerüst gab. Insbesondere seine Einordnung des terreur in die Geschichte der Revolution war für die moderaten Thermidorianer alarmierend: Für Lezay-Marnésia hatte der terreur durch seine Gewalttätigkeit und seinen Despotismus erfolgreich bewirkt, was keine andere historische Kraft bisher vollbracht hatte. Er habe ein „neues Volk“ geschaffen, indem er die alten Gewohnheiten (habitudes) in Stücke gerissen und das Volk über den Entwicklungsstand wilder Völker (état sauvage) wieder zum Naturzustand geführt habe (état naturel):

Achtzehn Monate Terror genügten, um dem Volk die Gebräuche mehrerer Jahrhunderte zu entreißen und ihm solche zu geben, die mehrere Jahrhunderte schwer hätten schaffen können. So also hat der Despotismus das Volk aus dem wilden Zustand befreit, wohin es durch die Revolution gelangt ist, und so hat seine Gewalt ein neues Volk geschaffen. Aus dem Despotismus konnte es in die Freiheit gelangen, was ihm nicht aus der Anarchie gelungen wäre (Lezay-Marnésia 1797: 45). ${ }^{20}$

Es ist also erkennbar, dass sich auch nach 1795 noch Stimmen erhoben, die dem Schock der Revolution positive Aspekte abgewinnen konnten. Sie blieben allerdings Ausnahmen, und die zentralen wissenschaftlichen Akteure innerhalb der Administration oder Regierungsinstitutionen nahmen eine deutlich andere Perspektive ein. Es war die Zeit, in der sich auch die Sciences morales et politiques für ein administrierendes Verständnis von Regieren einsetzten, das sich gegen eine allgemeine politische Praxis richtete (vgl. Wagner 1998).

\section{Das humanwissenschaftliche Gleichgewichtsmodell}

Für die Zeit nach dem terreurwar für die adminstrativ-politische Sphäre die Konsolidierung eines Verständnisses der Geschichtlichkeit der Revolution wichtig. Der Schriftsteller und Politiker Benjamin Constant (1767-1830), der nach dem terreur als Publizist die Ideale der Stabilität und Mäßigung vertrat, kritisierte deshalb das Buch Lezay-Marnésias harsch, vor allem dessen These, dass der terreur ein notwendiger Bestandteil der Revolution gewesen sei, weil so jedes Verbrechen gerechtfertigt werden könne (Con- 
stant 1797: 13). Für Constant war der terreur im Gegenteil eine Umkehrung aller natürlichen Werte. Zwar befürwortete er, einen Schleier über die Vergangenheit zu legen (ebd.: 14), es sei jedoch äußerst wichtig zu verhindern, dass sich das "System“ des terreur (im Unterschied zu seiner historischen Wirklichkeit in Gestalt einzelner „Terroristen“) wiederhole (ebd.: 16). Constant trat für eine Politik der durchgängigen Vigilanz ein, die aufmerksam auf die möglichen Verirrungen der Machthabenden achten sollte. Der terreur habe allenfalls dabei geholfen, die Hindernisse zu überwinden, die er selber geschaffen habe (ebd.: 28).

Die Humanwissenschaften, so wie sie sich in der „Klasse der moralischen und politischen Wissenschaften“ (Classe des sciences morales et politiques) des Institut national des sciences et des arts vereinten, teilten häufig die moderate Perspektive auf den terreur, die auch die Thermidorianer vertraten. Wie Martin Staum ausführlich analysiert hat, verfolgte diese im Jahr 1795 erstmalig in einer Regierungsinstitution verankerte Form humanwissenschaftlicher Forschung mehrheitlich das Ziel, durch Kontrolle der Leidenschaften die Verteidigung individueller Freiheit als Grundlage einer liberalistischen Technokratie und die Beruhigung der Temperamente die Revolution zu stabilisieren (Staum 1996: 4). In den sechs verschiedenen Sektionen der Klasse der moralischen und politischen Wissenschaften des Nationalinstituts - „Analyse der Wahrnehmungen und Ideen“ (analyse des sensations et des idées), "Moral“ (morale), "Sozialwissenschaft und Gesetzgebung“ (science sociale et législation), „politische Ökonomie“ (écononomie politique), Geschichte (histoire) und Geographie (géographie) fanden sich jeweils sechs ordentliche Mitglieder (membres résidants) und zusätzlich assoziierte Mitglieder zusammen, die mehrheitlich dieses Ziel verfolgten. Zudem hat diese zweite Klasse des heutigen Institut de France mehrere politische Akteure angezogen, die ihre Karriere während der Revolution begonnen hatten und die im Direktorium, dem Konsulat und dem empire weiterhin legislative und exekutive Funktionen ausübten (dazu Leterrier 1995: 13). Der napoleonischen Regierung erschien diese zweite Klasse ausreichend bedrohlich, um sie 1803 bei der Neustrukturierung des Nationalinstituts in vier Klassen aufzulösen. Die Forschung hat ausführlich gezeigt, dass sowohl die „Klasse der moralischen und politischen Wissenschaften" als auch der Kreis der mit den Humanwissenschaften verknüpften Akteure in der Zeit von 1795 bis 1799 in zweierlei Hinsicht richtungsweisend waren: zum einen durch die Konsolidierung eines wirksamen Vokabulars sowie einer Methodologie zur Analyse sozialer und politischer Phänomene; zum anderen durch direkte Interventionen in Gesetzgebung oder bei unmittelbaren politischen Aktionen (Staum 1996; Wokler 2000). Wie Jean Jamin und Georges Gusdorf gezeigt haben, gab es in der Zeit von 1795 bis 1799 kurzzeitig die Hoffnung, dass diese Humanwissenschaften, 
die auf der Wissenschaft von den „Ideologen“ verfochtenen sogenannten idéologie basierten, dabei helfen könnten, eine stabile Nation zu begründen. Insofern war ein Ziel dieser Gruppe von Intellektuellen, zentrale administrative und pädagogische Stellen zu besetzen, womit sie bis 1799 recht erfolgreich waren (vgl. Jamin 1982: 85), indem sie vor allem im Sektor der Instruction publique, wie etwa in der École normale (1795), der École centrale des travaux publics (seit 1795 École polytechnique), dem Conservatoire pour les machines (später Conservatoire des arts et métiers), dem Muséum national d'histoire naturelle und der Institution nationale des sourds-muets zentrale Stellen besetzten (Gusdorf 1978: 395-400). Im Anschluss an die politische Revolution hätten die idéologues, so Gusdorf, damit eine kulturelle Revolution auf den Weg gebracht und umgesetzt, deren Folgen bis in die Gegenwart andauerten (ebd.: 400). Neuere Forschungen bestätigen, dass diese Human- und die Sozialwissenschaften für eine Transformation intellektueller und kultureller Techniken Orientierungswissen bereitgestellt haben (vgl. Wokler 2000: 37). Zugleich habe, wie Peter Wagner herausgestrichen hat, die science sociale in der entstehenden Sphäre deliberativer Politik das überkommene Naturzustandsmodell (das sich selbst überlassenen Menschen Chaos und Unordnung zugeschrieben hatte) durch eine paradoxe Kopplung von politisch-sozialer Freiheit mit der basalen Vorhersagbarkeit und Steuerbarkeit von menschlichen Handlungen abgelöst (Wagner 1998: 243).

$\mathrm{Zu}$ ihrer projektierten Regierungskunst (art de gouverner) gehörte auch eine Analyse des gesellschaftlichen Entwicklungszustands sowie des Verhältnisses von sozialen Institutionen und dem Fortschritt der Aufklärung (progrès des lumières). Zu diesem Ziel trug Mediziner Pierre-Jean-Georges Cabanis (1757-1808) mit seinem viel gelesenen Buch Beziehungen des Physischen und des Moralischen beim Menschen (Rapports du physique et du moral de l'homme) von 1798 bei, das auf Vorlesungen vor der Sektion „Analyse der Sensationen und Ideen“ der Klasse der moralischen und politischen Wissenschaften beruhte. Er vertrat darin die These, dass gerade die Humanwissenschaften dabei helfen könnten, die Friktion zu glätten, die zwischen dem Fortschritt der Aufklärung, der sich im Zustand der esprits ausdrücke, und den Verhältnissen der sozialen und politischen Institutionen bestehe (Cabanis 1844 [1798]: 50). Dieser Unterschied in den Entwicklungszuständen habe die revolutionäre Gewalt überhaupt erst zu einer bestimmenden Kraft werden lassen. Schon Anfang der 1790er Jahre hatte Cabanis die These vertreten, dass der Ausbruch von Revolutionen hauptsächlich ein Werk der Menschen sei, wohingegen die "Natur" tendenziell auf einen Gleichgewichtszustand hinwirke, wenn sie nicht durch „schlechte Institutionen" (mauvaises institutions) daran gehindert würde (Cabanis 1823 [1791-1793]: 219). Die Wissenschaften vom Menschen hatten für Ca- 
banis die Aufgabe, darüber zu wachen, dass im Gesellschaftszustand die natürlichen Ungleichheiten, die „nützlich und gerecht“ seien, durch soziale Institutionen reguliert würden, die diesem natürlichen Zustand möglichst zuarbeiteten oder ihn sogar noch verbesserten (ebd.: 206). Besonders bei der „Klasse der Armen“ zeige sich die Macht schlechter Institutionen, denn die andauernde Ungerechtigkeit staue sich auf und entlade sich gerade in dieser wenig aufgeklärten Klasse als ungehinderte Wut der Leidenschaften:

[F]rüher oder später kommt der Augenblick, zu dem die Klasse der Armen erwacht. Und dieses Erwachen ist furchtbar. Wie könnte sie sich, unwissend und leidenschaftlich, ihrer Kraft angemessen bedienen? Indem sie ihre Kraft blind gegen alles wendet, was sie umgibt, wendet sie sie notwendigerweise auch gegen sich selbst. Ihre Anführer, wie weise und aufgeklärt sie auch sein mögen, sind beinahe immer gezwungen, sich ihren Leidenschaften zu beugen. (ebd.: 207-208). ${ }^{21}$

\section{Das Naturzustandsmodell}

Wie dargestellt war die Erziehung für die Humanwissenschaften ein zentrales Praxisfeld, besonders die Instruction publique, die das grundlegende Ziel der Aufklärung aller Bevölkerungsschichten und die damit verbundene Zirkulation von Wissen, Gütern und Meinungen ermöglichen sollte (vgl. Baczko 1982). Anders als für die Vigilanz der Thermidorianer konnte für den Erziehungsdiskurs (weniger für die entsprechenden Praktiken) eine Rückkehr zu einem Naturzustand mitunter als mögliche Lösung für die drohende Verführbarkeit des Volkes erscheinen. Besonders politisch moderate Deutungen während der verschiedenen Aufstände oder Massaker der Revolutionszeit nehmen diese Perspektive ein, die ein Hilfsmittel gegen die mutmaßlich unnatürlichen Leidenschaften des Volkes sein sollte.

Roch Ambroise Sicard (1742-1822), der Direktor der Institution nationale des sourds-muets, war einer der Priester, die den Eid auf die Zivilverfassung des Klerus, die Verstaatlichung der Kirche, verweigert hatten und deswegen während der sogenannten "Septembermassaker“ des Jahres 1792 mit anderen Adligen und Priestern inhaftiert wurde. Im Jahr 1796 publizierte er als glühender Verfechter der katholischen Fraktion in den Annales réligieuses einen Bericht über seine Erlebnisse. Das Volk erscheint darin als eine Masse, die einerseits von ihren wütenden Leidenschaften hingerissen wurde, andererseits fügsam genug war, sich von den Worten eines Führers lenken zu lassen. Sicard war sich sicher, dass die Massaker keineswegs spontan entstanden seien, sondern sich des „natürlicherweise irasziblen Charakters eines verirrten Mobs, dem man uns als seine grau- 
samsten Feinde denunzierte" bedient hätten (1835 [1797]: 101). ${ }^{22}$ Für diejenigen, die sowohl in der Verführbarkeit des Volkes als auch in der Möglichkeit der Rückkehr zu einem natürlichen Zustand Chancen erkannten, die politisch-sozialen Verhältnisse zu stabilisieren, erschien die Figur des sourd-muet - der ungefähr in dieser Zeit zusammen mit dem „Idioten“ zu einer administrativ-medizinischen Kategorie wurde - verheißungsvoll (dazu Buton 2009). Denn gerade ersterer erschien dieser moderaten Fraktion aufgrund seiner vermeintlichen Sprachlosigkeit und der daraus resultierenden Abwesenheit des sprachzeichenabhängigen Denkens als Hoffnung auf die Verwirklichung der politischen Utopie, Gesellschaft auf natürlichen Maßstäben neu aufbauen zu können. Die Deaf History hat gezeigt, wie die Konstruktion dieser administrativen, medizinischen und pädagogischen Subjektkonstruktion auf der Ausblendung der eigenen Kultur und Sprache gehörloser Personen beruhte (Quartararo 2008). Die Projektion dieser Subjektkategorie auf jene auch juristisch benachteiligte Gruppe (vgl. Rosenfeld 1997) konnte so zum Ausgangspunkt genommen werden, gerade hier den "neuen Menschen“ (l'homme nouveau) zu entdecken, der in der politischen Imagination vieler die Grundlage einer neuen Gesellschaft bilden sollte (vgl. Ozouf 1988b). Harlan Lane, François Buton und Anne Quartararo haben gezeigt, welche desaströsen Folgen die Konsolidierung dieser Kategorie für die Deaf Community hatte (Lane 1999 [1992]; Quartararo 2008; Buton 2009).

Das Werk, in dem Sicard seinen Praxisentwurf für eine auf sensualistischen Grundsätzen beruhende Erziehung darlegte, erschien im Jahr VIII (1799/1800) unter dem Titel Cours d'instruction d'un sourd-muet de naissance und sollte, so wollte es der Herausgeber, als „experimentelle Grammatik“ und „experimentelle Metaphysik“ verstanden werden (Vorwort in Sicard 1799/1800: x). Das Buch reagierte damit auf das Bedürfnis, die Natur des Menschen auf der Basis von natürlichen Fakten zu erforschen. Es war eine Antwort auf die Verunsicherung der philosophischen Konzeptionen des Naturzustandes, der als Grundlage der Intelligibilität politischer Prozesse ausgedient zu haben schien und dessen Eignung als adäquate Grundlage zur Formulierung sozialer und politischer Normen deshalb fraglich geworden war. Deshalb sollte der Naturzustand kein Thema der „Metaphysik“ mehr sein, sondern das Resultat beobachtender und experimentierender Wissenschaften. Diesen Standpunkt vertrat auch die nach Napoleon Bonapartes Staatsstreich gegründete „Gesellschaft der Menschenbeobachter" (Société des observateurs de l'homme, 1799-1804), deren Mitglied Joseph Marie de Gérando (1772-1842) meinte, die Revolution und der "Schock aller Meinungen“ (choc de toutes les opinions) habe es nötig gemacht, dass ein jeder die "Gesamtheit seiner Ideen über unsere Natur" (l'enceinte de ses idées sur notre nature) neu bewerten müsse 
(Gérando 1800: Band 3, 20). Deshalb stellte diese gelehrte Gesellschaft der "Zeit der Systeme“ (temps des systèmes) den „Weg der Beobachtung“ (route de l'observation) gegenüber (Gérando 1883 [1800]: 154). Die Beobachtung von Gehörlosen während ihrer Erziehung war die erste und aufsehenerregendste Umsetzung dieser Forderung nach einer empirischen Wissenschaft vom Menschen.

Sicards Cours d'instruction war somit nicht nur ein Unterrichtswerk, sondern auch ein herausragendes Beispiel der Humanwissenschaften mit ihren gesellschaftsstabilisierenden Zielen. Es steht für die Hoffnung, durch einen Rückgang auf den Naturzustand die Verirrungen sowohl der Revolution als auch des ancien régime heilen $\mathrm{zu}$ können. Die Vorstellung einer in der Konstruktion der Sprach- und Ideenlosigkeit seiner gehörlosen Schüler besonders sichtbaren und zu erhaltenden wohltätigen Natur durchzieht deshalb Sicards Forschungs- und Erziehungsepisoden. Sein berühmtester Schüler Jean Massieu (1772-1842) wurde im Narrativ des Cours d'instruction durch eine Serie von Interventionen dazu angeleitet, seinen unverstellten Blick auf die Natur in eine Ordnung von Zeichen zu übersetzen. Damit sollte er eine natürliche Ordnung beibehalten und eine Sprache generieren, die aufgrund ihrer „Natürlichkeit“ nicht täuschen und nicht verführen konnte. Ganz ausdrücklich wurde die von Sicard befürwortete Beobachtungskunst und seine als universell verstehbar präsentierte Version der "langue des signes“ als transformative Praxis aufgegriffen, die nicht nur die Gemeinschaft der Gehörlosen ihrer vermeintlichen Stummheit entheben sollte; in der weiteren politischen Diskussion wurde Sicards Vorschlag im Rahmen der Diskussion über die Verbesserung von Kommunikationsverfahren immer wieder als vorbildhaft aufgegriffen (vgl. Rosenfeld 2001). Sein Ansatz war der Versuch, die utopische Dimension der Revolution für die moralischen und politischen Wissenschaften zu übersetzen und im Rahmen eines Forschungs- und Erziehungsprogramms umzusetzen. In der langue des signes, der Zeichensprache, und den Individuen, die sie gebrauchten, erkannte Sicard die wohltätige Präsenz einer Natur, die nicht täuschen konnte und wollte:

[Die sourds-muets] benutzen ein Zeichen nur deshalb, weil sie eine Idee ausdrücken möchten. Die [Lautsprechenden] haben nur vage Begriffe, die von denjenigen übernommen werden, von denen sie ihre ersten Eindrücke empfangen, wo der Fehler sich häufig mit der Wahrheit mischt. Die letzteren [das heißt die sourds-muets] können überhaupt keinen Begriff von den Menschen übernehmen, die sie umgeben und zeigen demjenigen, der sie unterrichtet, eine immer neue Seele, oder sozusagen eine tabula rasa ohne Beimischung von heterogenen Ideen [...]. Er kann ihr die Lettern nach seinem Wunsch einprägen. Er 
kann schließlich in ihren Geist wie in einen reinen Behälter, den nichts hat verändern können, die genauesten Ideen dringen lassen ohne jede Beimischung von Fehlern. (Sicard 1799/1800: xxi) ${ }^{23}$

Hierbei wurde die Rückkehr zur „Natur“ durch geeignete Erziehungspraktiken als mögliche Utopie dargestellt, die für eine Zeit nicht nur im unmittelbaren Umfeld der Schule, sondern auch im politischen Diskurs aufgegriffen wurde. Die zugrundeliegende Hoffnung war, dass die Gewalt der Revolution kein Ausdruck einer schlechten Natur der Menschen gewesen sei, sondern ein Fehler, der durch eine bessere, natürlichere Moral und Form des Denkens und Sprechens behoben werden könne. Die Natur stellte sich hier, wie auch bei Cabanis, als sanfte Macht dar, die jede schockartige Veränderung unnötig machte. In der Anomie einer Humanwissenschaft, die sich weder auf Tradition noch auf irgendein Wesen des Menschen stützen wollte, erhofften die Akteure sich so in der Projektion auf einen vermeintlich empirisch auszumachenden natürlichen Zustand die Bestätigung ihres Wunsches nach einer stabilisierenden Referenz, die die Freiheit des Menschen, seine Tugend und Gesellschaftsfähigkeit garantierte. Durch die Vermeidung arbiträrer Sprache hofften diese Akteure den abus des mots und der damit einhergehenden Verwirrung der Ideen entrinnen zu können; auch Charles Maurice de Talleyrand-Périgord (1754-1838) glaubte, dass die von Sicard entwickelte "methodische Gestensprache“ die "Grammatik der Ideen" und nicht der Worte sei und dass damit die Gefahr des „Arbiträren“ gebannt werden könne (Talleyrand 1791: 100).

\section{Der therapeutische Schock}

Das Erscheinen eines sogenannten „Wilden“ in Paris und sein Transfer an die Institution nationale des sourds-muets im Jahr 1800 gab dem Mediziner Jean Itard (1774-1838) die Gelegenheit, Sicards Erziehung eines „Naturmenschen“ einer Prüfung zu unterziehen. Bei seinem Schüler handelte es sich um „Victor“, den sogenannten „Wilden von Aveyron“. Itard verfasste zwei Berichte über dessen Erziehung an den Innenminister, die sich von den vorigen Darstellungen solcher Begegnungen durch ihre Ausführlichkeit abheben; Adriana Benzaquén geht sogar davon aus, dass die Geschichte um Victor "most consequential in the history of the human sciences" sei (vgl. hierzu Benzaquén 2006: 144; vgl. auch Gineste 2004 [1993]). Der erste Bericht aus dem Jahr 1801 folgt der Überzeugung Sicards, dass Erziehung alles bewirken könne, und vertritt überwiegend die These, Victor vollständig wieder in die Gesellschaft einzugliedern. Hier 
ist Itard noch ein Vertreter jenes Naturzustandsutopismus', für den auch Sicard einstand:

In der wilden und am meisten vagabundierenden Horde wie auch in der zivilisiertesten Nation Europas ist der Mensch nichts anderes als was man aus ihm macht [...]. Er hat das vorzüglichste Vorrecht seiner Spezies genossen, die Begabung, seinen Verstand durch die Kraft der Imitation und den Einfluss der Gesellschaft zu entwickeln. (Itard 1894 [1801]: 1, Herv. L. S.) ${ }^{24}$

Auch ein undatierter Bericht aus den Archiven des heutigen Institut national de jeunes sourds de Paris hatte sich dieser Hoffnung auf die Wirkung der Imitation angeschlossen und glaubte, dass aus der imitativen Betätigung Victors einst eine „aktive Kraft der Vernunft“ entstehen würde, die ihn als Erfinder und Schöpfer über den Zustand eines bloßen Reproduzenten vorgefundener Strukturen heben würde, und Gérando erhoffte sich in einem Brief an Pierre-Louis Roederer (1754-1835) vom 14. März 1802, dass die „Wissenschaft vom menschlichen Verstand“ (science de l'entendement humain) erhebliche Fortschritte machen würde. ${ }^{25}$ Diese Hoffnung wird im späteren Bericht Itards aus dem Jahr 1806 zwar nicht vollständig aufgegeben, jedoch spezifiziert: Es gebe eine bestimmte Entwicklung, die Victor vorweisen könne, jedoch könne man seine Fortschritte nicht mit denen anderer Kinder vergleichen, sondern nur noch "mit sich selbst“ (Itard 1894 [1806]: 60). Mit diesem Bekenntnis zu einer Begrenzung des Vergleichsraums der Zivilisationsentwicklung war Itard Koproduzent eines Feldes von teilautonomen und teils sehr lokalen Zivilisationskonstellationen. Der universelle Maßstab der „Zivilisation“ wurde partikularisiert und ermöglichte nun von "der Zivilisation der Institution national des sourds-muets" zu sprechen, wie auch von den Fortschritten Victors, die nur für ihn selbst ein Maßstab sein sollten. Das ganze Verhältnis von Lehrer und Schüler bzw. Arzt und Patient stellte sich als Verhältnis unterschiedlicher Zivilisationen dar und nicht als Begegnung von Bürger_innen der einen universellen Zivilisation. Insofern wurde sie narrativ auch nicht als für beide gewinnbringend gefasst wie es bei Sicard und Massieu im Cours d'instruction der Fall war, sondern Victor erschien als Objekt von Itards Interventionen. Das emotionale Band, das auch bei Sicard als Mittler zwischen ihm und Massieu fungiert hatte, wurde bei Itard in diesem neuen Setting zum therapeutischen und epistemischen Instrument. Die Leidenschaften waren so nicht mehr nur die Voraussetzung und der Boden einer erfolgreichen Erziehung, sondern auch strategisch eingesetzte Requisiten. In folgender Passage nutzte Itard etwa Victors emotionalen Zustand, um eine schockartige Aufwallung herbeizuführen, die dazu dienen sollte, ein Erziehungshindernis zu überwinden: 
Ich näherte mich Victor, ich ließ ihn mitfühlende Worte hören, die ich in geeigneten Begriffen ausdrückte, um ihm den Sinn greifbar zu machen, und die ich mit umso verständlicheren Freundschaftsbezeugungen verknüpfte. Seine Tränen verdoppelten sich, begleitet durch Seufzer und Schluchzen. Während ich meine Liebkosungen meinerseits verdoppelte habe ich die Emotion zum höchsten Punkt getrieben und habe, wenn ich mich so ausdrücken darf, den moralischen Menschen bis zur letzten Faser erzittern lassen. (Itard 1894 [1806]: 80-81, Herv. L. S.) ${ }^{26}$

Ein von Thierry Gineste ediertes Manuskript Itards über nicht-organische Schädigungen des Intellekts (vésanies) aus dem Jahr 1802 zeigt, dass er schon hier in einem konzeptuellen Rahmen dachte, der temporale Unterschiede der Zivilisationsentwicklung unterschiedlicher Skalierung erfasste. Itard führte den Begriff des "Geistes der Gegenwart“ (esprit du temps actu$e l$ ) ein, vor dessen Hintergrund der graduelle Unterschied von Wahnsinn und Vernunft gemessen werden sollte, der sich in der Intensität der Leidenschaften ausdrückte (Itard 1802: 431). Diese Messung selber sei, so Itard, durch einen „unbestimmbaren Punkt" auf immer unsicher und drücke sich in „unzählbaren Variationen“ bei verschiedenen Individuen, Völkern und Jahrhunderten aus (ebd.: 437). Der Text forderte eine „Wissenschaft vom moralischen Menschen“, bei der ein Rest des Erziehungskonzepts von Sicard erhalten ist, indem der Arzt sich mit dem "moralischen Menschen“, den er überzeugen wolle, identifizieren müsse, „bis zu ihm herabsteigen“ solle und unglücklich wie er scheinen müsse, um eine Verbindung zu ihm zu erlangen (ebd.: 438). Die menschliche Leidenschaft war auch hier eine pathogene Kraft, die entweder als „nationale Manie“ (ebd.: 430) oder als Eigenschaft einzelner Tyrannen als lebensgefährlich erschien. Besonders die Tobsucht (fureur), die auch in "politischen Revolutionen“ als Eigenschaft einer ganzen Volksmasse zu beobachten sei (ebd.: 432), zeige als Charakterzug des Tyrannen ihre letale Wirkung:

Diese Leidenschaft hat die grausamsten und ausschweifendsten Tyrannen hervorgebracht: einen Nero, der sich mit dem Blut seiner ganzen Familie besudelt hat und der $\mathrm{zu}$ seinem Vergnügen Rom an allen vier Enden anzünden ließ; einen Caligula, der sich wünschte, dass das römische Volk nur einen einzigen Kopf haben möge, um ihn mit einem Schlag abzutrennen; einen Ludwig XI., einen Robespierre, der Freiheit und öffentliches Glück inmitten von Proskriptionen und Hinrichtungen schrie und tausende andere für ihre Grausamkeit berühmte Schurken, die viel weniger würdig sind, im Prunk der Geschichte zu erscheinen als in einer 
philosophischen Abhandlung über Geistesverirrungen. (Itard 1802: $432-433)^{27}$

Wie auch Cabanis vertrat Itard die These, dass Gesundheit in einem ausgeglichenen Verhältnis der Leidenschaften zur Umgebung bestand und übernahm sie auch für die moralische und intellektuelle Sphäre. Als Arzt an der Institution nationale des sourds-muets oblag Itard die Aufsicht über die Eignung der Bewerber_innen für Plätze an der Schule und auch hier argumentierte er im Rahmen teilweise autonomer Zivilationskonstellationen (INJS: Dossiert Itard). In seiner Abhandlung über die Krankheiten des Ohres und des Gehörs aus dem Jahr 1821 (Traité des maladies de l'oreille et de l'audition) erscheint die administrative Aufgabe als Einhegung und Erhaltung eines Gleichgewichtszustandes, der zwischen einem Zuviel und einem Zuwenig an „Zivilisation“ lag. Eine solche Ausgewogenheit war wiederum Teil eines insgesamten Zivilisationsfortschritts der „mimischen Gesellschaft":

Wenn man insgesamt unsere Taubstummen [sourds-muets] von heute mit den ersten Schülern vergleicht, die in derselben Institution ausgebildet wurden, mit derselben Methode und unter demselben Lehrer, so wird man zur Anerkennung einer Überlegenheit genötigt, die sie ihrem Vorteil schulden, später gekommen zu sein, zu einer fortgeschritteneren Periode der mimischen Gesellschaft. (Itard 1821: Band 2, $440-441)^{28}$

Auch andere Institutionen, die um 1800 geschaffen oder stark reformiert wurden - die Gefängnisse, die Krankenhäuser und Einrichtungen für Wahnsinnige - waren Orte, an denen ein kalkulierter therapeutischer Einsatz von starken Leidenschaften wieder möglich wurde, nachdem diese im politischen Diskurs der Jahre nach 1795 zunächst delegitimiert worden waren. So wurde die von Pinel aus England übernommene sogenannte "moralische Behandlung" (moral treatment, traitement moral) weiterentwickelt und popularisiert (vgl. Goldstein 1987: 65). Sie basierte auf der Überzeugung, dass bei Geisteskrankheiten auch Interventionen, die nicht auf einer direkten physischen Einwirkung beruhten, förderlich sein konnten. Pinel, der in Frankreich führende Mediziner auf diesem Gebiet, wählte gemeinsam mit dem Aufseher in der Anstalt für „wahnsinnige" Männer Bicêtre (erst am Ende des 19. Jahrhunderts regelmäßig „Psychiatrie“ genannt) Jean Baptiste Pussin (1745-1811) deshalb alle möglichen Formen der indirekten Behandlung durch Gespräche, Erschrecken, Theater, Musik etc. (vgl. ebd.: 72-77). Auch die Interventionen Itards, der bei Pinel Vorlesungen gehört hatte, stellten eine Weiterentwicklung dieses Konzepts dar. Dessen Kollege Jean Étienne Esquirol (1772-1840), der seit 1801 an der 
Salpêtrière, der Pariser Anstalt für „wahnsinnige“ Frauen, arbeitete, deren Leiter er neun Jahre später wurde, führte in seinem Text über die Leidenschaften 1805 den Begriff des „moralischen Schocks“ (sécousse morale) ein, der wie bei Petit den heilsamen Effekt von starken Emotionen begrifflich fassen sollte (Esquirol 1805: 82). Anders als bei Petit und genau wie bei Itard verschwanden jedoch bei Esquirol die heilsamen Wirkungen von politischen Schocks. Nur im Rahmen des kontrollierten Settings im Irrenhaus waren von Experten eingesetzte moralische Schocks sinnvoll, die eine "förderliche Krise" (crise favorable) stimulierten (ebd.: 12-13). Der moralische Schock, den der Experte herbeiführen sollte, war in seiner Heilwirkung dabei nicht mit dem pathogenen „politischen Schock“ zu vergleichen:

Indem die politischen Schocks alle Leidenschaften in Gang setzen, indem sie den künstlichen Leidenschaften Aufschwung geben, weil sie die boshaften Leidenschaften übersteigern, infolge der Bedürfnissteigerung bestimmter Individuen, indem sie anderen des Vermögens berauben, das für ihre Gewohnheiten nötig geworden ist, steigern die politischen Erschütterungen die Zahl der Wahnsinnigen. Dies hat man nach der Revolution in England beobachtet, dies beobachtet man auch in Frankreich nach unserem revolutionären Sturm. (ebd.: 15) ${ }^{29}$

Anders als Itards Bekenntnis zu nivellierten Gleichzeitigkeitsregimes ist Esquirol hier noch - unter anderem unter Bezug auf Cabanis - der Überzeugung, dass Wahnsinn in vielen Fällen heilbar sei, indem Wahnsinnigen die "schädliche Kette von Ideen“ (chaîne vicieuse des idées) und die "dichte Wolke, die die Intelligenz verdunkelt" (nuage épais qui obscurcissait l'intelligence) besonders bei akuten geistigen Krankheiten durch einen Schock vertrieben würden, der es ihnen ermöglichen würde „sich selbst zu überwinden" (à se surmonter lui-même) (ebd.: 85).

Ohne die weitere Diskussion von therapeutischen gegenüber revolutionären Schocks hier im Einzelnen verfolgen zu können, möchte ich noch als weiteres Beispiel auf eine Preisfrage der Société libre des sciences physiques \& médicales in Lüttich zum "Einfluss der Leidenschaften auf die Produktion von Krankheiten" (De l'influence des passions sur la production des maladies) aus dem Jahr 1809 verweisen, die davon ausging, dass die Medizin sich bisher zu wenig mit dem Einsatz der Leidenschaften befasst habe. Auch hier wird Vorsicht angeraten und durch medizinische Eingriffe eine Harmonie zwischen "moralischem" und "physischem" Wesen angestrebt (Sauveur 1809: 34). Eine Einsendung eines Mediziners aus Grenoble, Hippolyte Bilon (1780-1824), betont dabei vor allem die desaströsen Wirkungen der revolutionären Wirren auf die Menschen: Anomalien der Nerven entstünden, Krankheiten aus Schwäche, chronische Krankheiten wie Asthma oder Schwindsucht (Bilon 1809: 352). Bezugnehmend auf Pe- 
tit, der auch die positiven Effekte hervorgehoben hatte, glaubte Bilon nicht mehr an die heilsame Kraft der Revolution: „Revolutionen steigern die gleichen Leidenschaften, die ihre Ursache waren" (les révolutions exaltent ces mêmes passions qui furent leur cause, Übersetzung L. S., ebd.: 353).

Esquirols und auch Pinels Hoffnung auf die Heilbarkeit des Wahnsinns stützte sich auf ein Konzept des Geistes, wie sie es im Wesentlichen von Condillac übernommen und angepasst hatten (vgl. hierzu Paradis 1993). Zwar stellte schon Pinel fest, dass die Beschreibung der menschlichen Leidenschaften bei Condillac grundlegend erneuert und durch medizinische Beobachtungstechniken gestützt werden müsste (Pinel 1798: 24), dennoch gingen sowohl er als auch Esquirol weiterhin davon aus, dass die Heilung des Wahnsinns als eine Rückkehr zu einem rationalen Selbst dargestellt werden könne, wie Gladys Swain und Marcel Gauchet ausführlich analysiert haben: Ein Gespräch mit dem Wahnsinnigen mache bei Pinel überhaupt nur deshalb einen Sinn, weil der Wahnsinn selber immer zugleich total sei - indem er alle Verstandeskräfte störe - aber auch partiell weil hinter dem Wahnsinn unbeschadet ein rationales Selbst weiterbestehe, zu dem der Wahnsinnige zurückkehren könne (Gauchet 1997: 31; Swain 1997: 71). Dieses rationale Selbst blieb im Wesentlichen ein universalistischer und überall gleich funktionierender, vorwiegend passiver und stabiler Transformator von Sinneswahrnehmung. Seine epistemische Grundstruktur wandelte sich auch nicht, als sich die Bewertung anarchischer Leidenschaften inmitten der Revolutionswirren veränderte. Doch in der Praxis des kontrollierten moralischen Schocks kündigt sich eine veränderte Haltung zum Inhalt und zur Struktur des Geistes an, die Doris Kaufmann unter der Überschrift des Subjekts im „bürgerlichen Gefährdungsdiskurs“ verhandelt (Kaufmann 1995: 18): ein fragiles Selbst, das nach Gauchet seit Esquirol den Konflikt mit dem Wahn ins Innere des moi verlagert habe (Gauchet 1997: 35). Insofern erscheint es nur folgerichtig, dass es auch Esquirol war, der dem „moralischen Schock“ einen Begriff gegeben hat.

Viele Diskussionen über diese neue Subjektkonstitution formatierten sich durch eine Neudeutung des zentralen Geistesvermögens der „Aufmerksamkeit“ (attention), dem geistigen Pendant zur physischen „Sensibilität", und deshalb wurde auch die Aufmerksamkeit ein zentraler Gegenstand von therapeutischen und edukativen Maßnahmen. Für Esquirol waren alle Geisteskrankheiten letztlich auf eine Schädigung der Aufmerksamkeit zurückzuführen, wie er hier mehr als dreißig Jahre später in seinem umfassenden Werk über Geisteskrankheiten verdeutlichte: „Der Wahnsinnige hat nicht mehr das Vermögen, seine Aufmerksamkeit zu bestimmen und zu lenken. Dieser Verlust ist der ursprüngliche Grund aller seiner Fehler" (Esquirol 1838: 20). ${ }^{30}$ Noch bei Sicard war die Aufmerksamkeit eine Art Naturereignis gewesen, das nicht weiter erklärt worden war, eine Leerstelle 
in dem ansonsten an Definitionen überdeterminierten System der körperlichen und seelischen Wahrnehmung und des Denkens (Sicard 1799/1800: 374).

Die Kritik an einer orthodoxen Auslegung Condillacs um 1800 richtete sich deshalb darauf, die Aufmerksamkeit als möglichen Angriffspunkt zu wählen und hob hervor, dass sie, passiv gedeutet, der kreativen und bisweilen rätselhaften Autonomie des Menschen zu wenig Platz einräume. In den ersten Zeilen von Des signes definierte Gérando so das Verb „denken“ analog zu „handeln“: „Denken [penser] ist für den Geist, was handeln [agir] für den Körper ist" (Penser est à l'esprit, ce qu'agir est au corps) (Gérando 1800: Bd. 1, S. 2). So suchten auch Itard und die sich auf ihn beziehenden Ärzte, die „Aktivität" und das „Genie“ ihrer Zöglinge zu stimulieren. Dieses „Ich“, so Kaufmann mit Bezug auf die deutschsprachige Tradition, wurde als fragiler und gefährdeter Besitz des bürgerlichen Subjekts auch aus Sicht der psychiatrischen Praxis - etwa bei Wilhelm Griesinger (1817-1868) nur ungern voreilig schockartigen, „perturbativen“ Methoden ausgesetzt (Griesinger 1861: 471). So wie die Thermidorianer einen Gefährdungsdiskurs über die politische Fragilität des Volkes instituierten, hatten die an der Praxis des therapeutischen Schocks arbeitenden Mediziner unter direktem Bezug auf dieselbe Erfahrung des terreur an der Produktion eines fragilen moi gearbeitet.

\section{Schluss}

Alle vorgestellten Formen von Steuerungstechniken - seien es die medizinischen Techniken in den spezialisierten Einrichtungen oder die Projekte von Regierungstechniken in Form einer sozialen Kunst oder einer Politikwissenschaft - lehnten nach der Zeit des terreur explizit oder implizit die Vorstellung eines heilsamen, kollektiven und revolutionären Schocks ab. Stattdessen haben sich um die reformierte Version eines Condillacschen Subjekts, das durch eine autonome Sphäre der Innerlichkeit gekennzeichnet war, und das insbesondere in der Diskussion des Bürgertums als unantastbarer Besitz gehandelt wurde (Kaufmann 1995: 17, 174; Goldstein 2005: 162-165), eine Reihe von therapeutischen und epistemischen Techniken gebildet, die den Schock als mögliches Mittel betrachteten, sofern er durch medizinische Expertise legitimiert war. Wie die Forschung gezeigt hat, reagierten sie damit auf die Herausforderung der Amerikanischen und Französischen Revolution, zugleich eine liberale Sphäre freier und gleicher Individuen geschaffen zu haben und die Idee, dass deren Verhalten steuerbar und vorhersehbar sei (Wagner 1998: 247), indem sie ein Individuum 
schufen, das einen Bereich besaß, der rätselhaft und unzugänglich war. Der in der sozialistischen Tradition bei Saint-Simon oder Engels aufgegriffene Begriff von Planbarkeit (Wokler 2000) wurde in der bürgerlichen Deutung durch dieses neue Subjektverständnis aus der Kritik an einem orthodoxen Condillacismus komplementiert. Dieses rätselhafte Innere ermöglichte andererseits Subjekte, denen ein immer fixeres Wesen zugeschrieben wurde (Chappey 2017: 220). Erschütterungen, Schocks erschienen hier nicht mehr als Utopie der Befreiung, weder von Einzelnen und nicht des Volkes, sondern als gefährliches und letztes Mittel, das nur in Ausnahmefällen möglicherweise Besserung versprach. Dem prekären inneren Gleichgewicht des bürgerlichen Selbst entsprach so ein als prekär wahrgenommenes Gleichgewicht der politischen Sphäre, wobei immer zwischen dem Recht von Eliten zur Kontrolle von Individuen und dem Recht dieser Individuen auf die Unantastbarkeit ihres moi balanciert werden musste.

Die thematisierten medizinischen und pädagogischen Techniken wurden vor dem Hintergrund einer durchgängigen Auseinandersetzung mit den Leidenschaften während der Französischen Revolution und besonders während der Zeit des terreur erarbeitet. Es haben sich hierbei verschiedene Modelle gebildet, die Leidenschaften des Volkes und von Individuen medizinisch-therapeutisch zu bewerten und schließlich einzusetzen: (a) Das erste Modell wurde als die utopisch-revolutionäre Variante gekennzeichnet. Die Leidenschaften des Volkes wurden hier als notwendiges Mittel und logische Folge der legitimen Forderung nach Anerkennung von Rechten des Volkes (Bürgerrechte, Menschenrechte) betrachtet und als Mittel, die alten Zustände, Gewohnheiten und Sitten radikal abzuschaffen. (b) Das zweite humanwissenschaftliche Modell sah in den ausbrechenden Leidenschaften des Volkes auch ein Anzeichen für die Historizität von Aufklärung und sozialen Institutionen, negierte aber die Notwendigkeit dieses Ausbruchs. Es wurde gezeigt, wie Cabanis davon ausging, dass gerade $\mathrm{Hu}-$ manwissenschaften dazu beitragen könnten, den Anachronismus zwischen dem Zustand der Geister und dem der sozialen Institutionen zu erkennen und geeignete Gegenmaßnahmen zu identifizieren. (c) Im Rahmen eines dritten, unhistorischen Modells war der Schock der Revolution und der Ausbruch von terroristischen Emotionen eine Abirrung, deren Wiederkehr nur durch die Erschließung eines empirisch identifizierten Naturzustands verhindert werden konnte. In diesem Naturzustand wurden die Ideale von Harmonie, Mäßigung und auch einer natürlichen Religion aufgefunden, die durch den zivilisatorischen Luxus überfrachtet und unsichtbar geworden waren. (d) Das vierte Modell unterschied zwischen einem politischen und einem therapeutischen Schock. Ersterer war schädlich, letzterer konnte heilsam sein, wenn er von Experten in kontrollierten Kontexten eingesetzt wurde. Der heilsame Schock war hierbei in jedem Fall eine in spezialisier- 
ten Institutionen im Rahmen eines hierarchischen Verhältnisses zwischen Arzt und Patient_in eingesetzte, kontrollierte und wissensbasierte Technik. Auch wenn sich im Lauf des 19. Jahrhunderts sicher noch weitere Deutungen bildeten, die Erfahrungen revolutionärer Gewalt verständlich machten (wie das epidemische Modell (Rosen 1959: 15)), blieben, so die These, die vier Modelle, das utopisch-revolutionäre, das moderierend-humanwissenschaftliche, das naturzustandstheoretische und das therapeutische mögliche Optionen.

Zweitens wurde aus der Perspektive des Schocks gezeigt, dass sich das Verhältnis zwischen Forschern_innen/Ärzt_innen und menschlichen Forschungsobjekten/Patient_innen veränderte. Im Rahmen der Steuerungstechniken, die von den vorgestellten Akteuren projektiert und partiell umgesetzt wurden, wurde eine zumindest potentiell gleichberechtigte Beziehung zwischen Forscher_in und menschlichem Forschungsgegenstand von einer Beziehung abgelöst, die im menschlichen Forschungsobjekt ein Individuum erkannte, das durch eine transformierte Sphäre der Innerlichkeit charakterisiert wurde. Bei Gérando und Maine de Biran als moi konzeptionalisiert, bestimmte sich diese Sphäre durch ihre Unsichtbarkeit und Unzugänglichkeit. Der Schock, der in solchen experimentellen Settings eingesetzt wurde, richtete sich gegen die politische Deutung des Schocks und zielte auf die Sichtbarmachung und Therapie von Aspekten dieses moi. Andere Akteure, Philosophen wie Victor Cousin, Psychiater wie Félix Voisin (1794-1872), Verwalter wie Gérando, haben in Weiterentwicklung von oder Abgrenzung zu früheren Ansätzen Techniken der Sichtbarmachung entwickelt, die sich um die menschliche Innerlichkeit gruppierten (vgl. Voisin 1826). Der Vorbehalt gegenüber politischen Revolutionen und den mit ihnen verknüpften Leidenschaften sollte im psychiatrischen Diskurs weiterbestehen, wie etwa bei Jean-Étienne Georget (1795-1828), einem Schüler von Esquirol: „Der Wahnsinn ist ebenfalls besonders in den freien Ländern verbreitet, bei den Völkern, die durch Gruppen und Parteien erschüttert werden, die gewalttätigen politischen Erschütterungen ausgesetzt sind, die alle Elemente der Gesellschaft umstürzen [...] in einem Wort: Diese Krankheit wird durch die Umstände erzeugt und vermehrt, die die Aufmerksamkeit erregen, den Geist (esprit) aktivieren, und alle Leidenschaften des Menschen ins Spiel bringen" (Georget 1823: 8). ${ }^{31}$

\section{Danksagung}

Der Aufsatz wäre ohne die Diskussionen mit Moritz Epple und Martin Herrnstadt im Forschungsprojekt „Epistemologien des Wissens vom Men- 
schen“ in Frankfurt nicht möglich gewesen. Dafür möchte ich mich herzlich bedanken.

\section{Anmerkungen}

1 "Quel vaste \& mobile tableau des effets variés des passions humaines, présente le réveil d'un grand peuple, qui sort d'une longe léthargie!" (Übersetzung L. S.), Tableau des opérations de l'Assemblée nationale d'après le Journal de Paris (Lausanne: Chez Hignou \& Comp. 1789), Bd. 2, S. 227. Ich werde in diesem Aufsatz durchgängig die Übersetzung "Leidenschaft" des französischen Wortes „passion“ verwenden.

2 Dies gilt auch, wenn Dora Weiner lange daran gearbeitet hat, den „Mythos" Pinel zu kritisieren, der diesen als „Befreier" darstellt und bis in die heutige Zeit fortwirk (Weiner 2008). Zur Geschichte psychiatrischer Behandlungsmethoden, vgl. klassisch Goldstein (1987). Die Rolle des Schocks in der Französischen Revolution als Vorgeschichte von PTBS wurde von Steinberg (2015) beleuchtet. Vgl. allgemein zur Psychiatriegeschichte in der revolutionären Phase Lerner (1997), zur Rolle der Leidenschaften Pigeaud (1980). Vgl. zur medizinischen Deutung der Französischen Revolution und zur Rolle der Psychiatrie: Gelbart (1989).

3 „le corps dépérissait dans l'inaction par le progrès de la mollesse \& du luxe, \& l'activité inquiète de l'esprit humain ne pouvait plus être soutenue“, „la plus violente explosion de patriotisme \& de courage“, „,Je me porte mieux depuis la révolution" (Übersetzung L. S.), Tableau des opérations de l'Assemblée nationale d'après le Journal de Paris (Lausanne: Chez Hignou \& Comp. 1789), Bd. 2, S. 227.

4 Ich werde das generische Maskulinum verwenden, sofern sich in meinem Untersuchungszeitraum Frauen nicht beteiligt haben oder nicht beteiligen konnten.

5 Zu diesem sehr weit verbreiteten Konzept vgl. beispielsweise Duchet (1995 [1971]).

6 Dies war eine weit verbreitete Argumentationsstrategie in Bezug auf die chinesische Despotie; vgl. dazu Roberts (1997) und Guy (1963).

7 Dies zeigt sich bei Destutt de Tracy, vgl. dazu Messling (2013).

8 „sa bouche est impure, son haleine fétide; jamais il ne lave son corps; jamais il ne lui fait recevoir immédiatement l'impression salutaire de l'air libre“ (Übersetzung L. S.).

9 „Les esprits [...] ne sont pas encore disposés à vous entendre; vous allez choquer beaucoup du monde? Il le faut ainsi: la vérité la plus utile à publier, n'est pas celle dont on étoit déjà assez voisin, ce n'est pas celle que l'on étoit déjà près d'accueillir. Non, c'est précisément parce qu'elle va irriter plus de préjugés et plus d'intérêts personnels, qu'il est plus nécessaire de la répandre" (Sieyès 1789: 174).

10 Tableau des opérations de l'Assemblée nationale d'après le Journal de Paris (Lausanne: Chez Hignou \& Comp., 1789), Bd. 2, S. 227.

11 Ich stütze mich hier auf Forschungen, die diesen allgemeinen Diskurs umfassend dargestellt haben: Baczko (1994 [1989]); Jainchill (2008). Quinlan hat demgegenüber darauf hingewiesen, dass die Rolle der Ärzte im Diskurs über die „moralische Regeneration“ nach dem terreur vernachlässigt werde, vgl. Quinlan (2004: 140).

12 Es existierte eine Debatte darüber, ob Condillac tatsächlich diese These in ihrer starken Form vertreten habe, was häufig aus dem Traité de sensations, der Abhandlung über Sinneswahrnehmungen, abgeleitet wird. Einflussreich hat Hans Aarsleff in Frage aufgeworfen, ob Condillac hier wirklich die Behauptung aufgestellt habe, dass alle menschlichen Vermögen aus der Sinneswahrnehmung zu erklären seien (Aarsleff 1982: 153 f.). Meines Erachtens nach hat Condillac in diesem Text in der Tat diese These in ihrer starken Form vertreten, für die Zwecke dieses Aufsatzes genügt es aber, auf die sehr wichtige Rolle der Sinneswahrnehmung zu verweisen, die sie auch in Aarsleffs Deutung einnimmt. 
$13 \mathrm{Zu}$ den Festen vgl. Ozouf (1988a); zur Diskussion der Straßennamen vgl. Ferguson (1997).

14 „Un changement brusque et complet“. Die Übersetzung ist aus (Destutt de Tracy 2016 [1803]: 204 f.) entnommen.

15 „dans la république, toutes les têtes sont pour ainsi dire imprégnées de poudre, et que les moindres étincelles que jetteraient à droite ou à gauche les discussions de la Convention mettraient inévitablement le feu dans quelque partie de la république“ (Übersetzung L. S.).

16 „le dérangement de toutes les idées, le renversement de toutes les affections“; „tremblement extérieur qui affecte les fibres les plus cachées, qui dégrade l'homme et l'assimile à la brute“; „ébranlement de toutes les forces physiques, la commmotion de toutes les facultés morales", (Übersetzung L. S.).

17 „Le despotisme artificieusement caché sous le masque des vertus et de l'hypocrisie n'en imposera plus ... Les monstres! ... il ne prenaient votre langage que pour mieux nous étouffer" (Übersetzung L. S.).

18 Letzterer ist schon deutlich älter; es findet sich beispielsweise auch eine medizinische Tradition der Reflexion über die Leidenschaft des terroris, als perturbatio animi etwa bei Johannes Oosterdyk Schacht zu Beginn des 18. Jahrhunderts. Ein durch Eintauchen in kaltes Wasser erzeugter terrore wäre nach Schacht beispielsweise - eine entsprechend robuste Natur des Patienten vorausgesetzt - bisweilen in der Lage, Tollwut zu heilen (Schacht 1733: 34-35). Auch bei Scheidemantel finden sich entsprechende Überlegungen (Scheidemantel 1787).

19 „Les révolutions sont au corps politique qu'elles agitent, ce que sont au corps humain altéré les médicamens qui doivent y rétablir l'harmonie. Dans l'un comme dans l'autre, le premier effet est un désordre, la première sensation une douleur" (Übersetzung L. S.).

20 „Dix-huit mois de terreur suffirent pour enlever au peuples des usages de plusieurs siècles, et pour lui en donner que plusieurs siècles auroient eu peine à établir. Voilà comment ce despotisme le tira de l'état sauvage où l'avoit fait passer la révolution, et comment sa violence en fit un peuple neuf. Du despotisme, il put passer à la liberté, il ne l'auroit pas pu de l'anarchie" (Übersetzung L. S.).

21 „Mais, tôt ou tard, le moment de son [gemeint ist die „classe pauvre"] réveil arrive; et ce réveil est terrible. Ignorante et passionnée, comment pourra-t-elle se servir convenablement de sa force? En l'exerçant aveuglément contre tout ce qui l'entoure, elle la tourne nécessairement contre elle-même. Ses chefs, quelque sages et éclairés qu'on les suppose, sont presque toujours forcés de se plier à ses passions“ (Übersetzung L. S.).

22 „caractère naturellement irascible d'une populace égarée à laquelle on nous dénonçait comme ses plus cruels ennemis" (Übersetzung L. S.).

23 „Les premiers [die Lautsprechenden, L. S.] prononcent long-temps des mots sans y attacher aucune idée, et en conservent souvent l'habitude pendant le reste de leur vie; les seconds [die sourds-muets, L. S.] n'employent un signe, que parce qu'ils ont une idée à exprimer. Les premiers n'ont que des notions vagues, recueillies parmi ceux de qui ils reçoivent les premières impressions, où l'erreur se mêle souvent avec la vérité; les seconds ne pouvant recevoir aucune notion de ceux qui les entourent, présentent à celui qui les instruit une ame toujours neuve, ou, comme on dit, une table rase, sans mélange d'idées hétérogènes [...]. Il peut y tracer, à son aise, les caractères qu'il veut y imprimer. Il peut enfin faire entrer dans leur esprit, comme dans un vase pur que rien n'a pu altérer, les idées les plus justes, sans aucun mélange d'erreur" (Übersetzung L. S.).

24 „Dans la horde sauvage la plus vagabonde comme dans la nation d'Europe la plus civilisée, l'homme n'est que ce qu'on le fait être; [... il a joui de la plus belle prérogative de son espèce, la susceptibilité de développer son entendement par la force de l'imitation et l'influence de la société" (Übersetzung L. S.).

25 Das vollständige Zitat lautet: „on ne croit pas d'abord y remarquer un pas qu'un doive attribuer à la force active de la raison. mais si ce victor d'aujourd'hui, imitant, par chacun des sons exercés, les actions dont on le rend temoin, est comparé à ce jeune Sauvage 
qui n'avoit de l'espece humaine que la structure mécanique, s'il est permis de présumer le futur par le présent, et de supposer que la marche du plus connu au moins connu est toujours sure, ne peut on pas espérer que celui qui imite des lignes et des cercles, pourra ecrire un jour [...] [?]“ (INJS: Dossier Itard, Chemise „sauvage“). Der zitierte Brief an Roederer findet sich in (AN: 29AP/10).

26 „Je me rapprochai de Victor; je lui fis entendre des paroles affectueuses, que je prononçai dans des termes propres à lui en faire saisir le sens, et que j'accompagnai de témoignages d'amitié plus intelligibles encore. Ses pleurs redoublèrent, accompagnées de soupirs et de sanglots; tandis que redoublant moi-même de caresses, je portai l'émotion au plus haut point, et faisais, si je puis m'exprimer, frémir jusqu'à la dernière fibre sensible l'homme moral“ (Übersetzung L. S.).

27 „Cette passion produisit les tyrans les plus cruels et les plus extravagants: un Néron qui se souille du sang de toute sa famille, faisant mettre pour son plaisir le feu aux quatre coins de Rome: un Caligula qui désirait que le peuple romain n'eût qu'une seule tête pour l'abattre d'un seul coup: un Louis XI., un Robespierre criant liberté et bonheur publics au milieu des proscriptions et des supplices, et mille autre scélérats fameux pour leur cruauté, véritables maniaques bien moins dignes de figurer dans les fastes de l'histoire que dans un traité philosophique de l'aliénation mentale“ (Übersetzung L. S.).

28 „En comparant collectivement nos sourds-muets d'aujourd'hui, aux premiers élèves formés dans la même Institution, par la même méthode, sous le même maître, on est conduit à reconnaître une supériorité dont ils ne peuvent être redevables qu'à l'avantage d'être venus plus tard, à une période plus avancé de la société mimique“ (Übersetzung L. S.).

29 „Les sécousses politiques en mettant en jeu toutes les passions, en donnant plus d'essor aux passions factices, en exagérant les passions haineuses, en multipliant les besoins de certains individus, en privant les autres d'une fortune devenue nécessaire à leurs habitudes, les commotions politiques augmentent le nombre des aliénés; c'est ce qu'on a observé après la révolution d'Angleterre; c'est ce qu'on observe en France depuis notre tourmente révolutionnaire" (Übersetzung L. S.).

30 „l'aliéné ne jouit plus de la faculté de fixer, de diriger son attention; cette privation est la cause primitive de toutes ses erreurs" (Übersetzung L. S.).

31 „Aussi la folie est-elle particulièrement commune dans les pays libres, chez les peuples agités par les factions et les partis, soumis à des commotions politiques violentes qui bouleversent tous les élémens de la société[...] en un mot, cette maladie naît et se multiplie avec les circonstances qui excitent vivement l'attention, activent l'esprit, et mettent en jeu toutes les passions de l'homme" (Übersetzung L. S.).

\section{Ungedruckte Quellen}

- Institut national de jeunes sourds de Paris (= INJS)

- Dossier zu Jean Itard und seiner Erziehung des „Wilden von Aveyron“: Dossier Itard, Chemise "sauvage“

- Archives nationales, Paris (= AN)

- Unterlagen zur Institution nationale des sourds-muets: FN/15/2584 


\section{Literatur}

Aarsleff, Hans 1982. From Locke to Saussure. Essays on the Study of Language and Intellectual History. London: Athlone.

Baczko, Bronisław 1982. Introduction. In: Bronisław Baczko (Hg.). Une éducation pour la démocratie. Paris: Éditions Granier Frères: 9-58.

Baczko, Bronisław 1994 [1989]. Ending the Terror. The French Revolution after Robespierre. Cambridge: Cambridge University Press.

Barère, Betrand 1794. Rede vor der Convention nationale. Moniteur (315, 15 Thermidor an II/02.08.1794): 369-372.

Benzaquén, Adriana 2006. Encounters with Wild Children. Temptation and Disappointment in the Study of Human Nature. Montreal \& Kingston/London/Ithaca: McGill-Queen's University Press.

Bilon, Hyppolite 1809. De l'influence des passions sur la production des maladies. In: Société libre des sciences physiques \& médicales de Liège (Hg.). De l'influence des passions sur la production des maladies. Lüttich: Chez J. Desoer: 291-354.

Buton, François 2009. L'administration des faveurs. L'État, les sourds et les aveugles (1789-1885). Rennes: Presses Univ. de Rennes.

Cabanis, Pierre-Jean-Georges 1844 [1798]. Rapports du physique et du moral de l'homme. Paris: J.-B. Baillière.

Cabanis, Pierre-Jean-Georges 1823 [1791-1793]. Quelques principes et quelques vues sur les secours publics. In: ders., Oeuvres complètes. Band 2. Paris: Bossange Frères, Didot.

Chappey, Jean-Luc 2002. La Société des observateurs de l'homme: (1799-1804). Des anthropologues au temps de Bonaparte. Paris: Société des Études Robespierristes.

Chappey, Jean-Luc 2017. Sauvagerie et civilisation. Une histoire politique de Victor de l'Aveyron. Paris: Fayard.

Constant, Benjamin 1797. Des effets de la terreur. s.1.

David, Pierre 1801. Epître à l'abbé Sicard, sur les mots avec lesquels on nous a gouverné pendant la Révolution. Paris: Chez les Marchands de Nouveautés.

Destutt de Tracy, Antoine Louis Claude 1803. Élémens d'idéologie. Seconde partie: grammaire. Paris: Chez Courcier.

Destutt de Tracy, Antoine Louis Claude 2016 [1803]. Grammatik. Stuttgart-Bad Cannstadt: frommann-holzboog. Übersetzung auf der Grundlage Claus Sonnenschein-Werners, bearbeitet von Hans Jörg Sandkühler.

Duchet, Michèle 1995 [1971]. Anthropologie et histoire au siècle des lumières. Paris: Albin Michel.

Esquirol, Jean Étienne Dominique 1805. Des passions considérées comme causes, symptômes et moyens curatifs de l'aliénation mentale. Paris: Didot jeune.

Esquirol, Jean Étienne Dominique 1838. Des maladies mentales considérées sous les rapports médical, hygiénique et médico-légal. Paris: Baillière.

Ferguson, Priscilla 1997. Paris as Revolution. Writing the Nineteenth-Century City. Berkeley: University of California Press.

Foucault, Michel 1969 [1961]. Wahnsinn und Gesellschaft. Frankfurt am Main: Suhrkamp.

Foucault, Michel 1974 [1966]. Die Ordnung der Dinge. Eine Archäologie der Humanwissenschaften. Frankfurt am Main: Suhrkamp.

Foucault, Michel 1976 [1963]. Die Geburt der Klinik. Eine Archäologie des ärztlichen Blicks. Frankfurt am Main/Berlin/Wien: Ullstein.

Gauchet, Marcel 1997. De Pinel à Freud. In: Gladys Swain. Le sujet de la folie. Naissance de la psychiatrie. Paris: Calmann-Lévy: 7-57.

Gelbart, Nina Rattner 1989. The French Revolution as Medical Event. The Journalistic Gaze. History of European Ideas (10/4): 417-427.

Georget, Étienne-Jean 1823. De la folie ou aliénation mentale. Paris: De l'imprimerie de Rignoux.

Gérando, Joseph-Marie 1800. Des signes et de l'art de penser considérés dans leur rapports mutuels. 3 Bde. Paris: Chez Goujon u.a. 
Gérando, Joseph Marie 1883 [1800]. Considérations sur les diverses méthodes à suivre dans l'observation des peuples sauvages. Revue d'anthropologie (12): 153-182.

Gineste, Thierry 2004 [1993]. Victor de l'Aveyron. Dernier enfant sauvage, premier enfant fou. Paris: Hachette Littératures.

Goldstein, Jan 1987. Console and Classify. The French Psychiatric Profession in the Nineteenth Century. New York u.a.: Cambridge University Press.

Goldstein, Jan 2005. The Post-Revolutionary Self. Politics and Psyche in France, 1750-1850. Cambridge (Mass.): Harvard University Press.

Griesinger, Wilhelm 1861. Die Pathologie und Therapie der psychischen Krankheiten. Für Arzte und Studierende. Stuttgart: Adolph Krabbe.

Gusdorf, Georges 1960. Introduction aux Sciences Humaines. Essai critique sur leurs origines et leur développement. Paris: Les Belles Lettres.

Gusdorf, Georges 1978. La conscience révolutionnaire. Les idéologues. Paris: Payot.

Guy, Basil 1963. The French Image of China before and after Voltaire. Genf: Institut et Musée Voltaire.

Itard, Jean 1821. Traité des Maladies de l'Oreille et de l'Audition. 2 Bde. Paris: Chez Méquignon-Marvis.

Itard, Jean 1802. Vésanies. In: Thierry Gineste 2004 [1993]. Victor de l'Aveyron. Dernier enfant sauvage, premier enfant fou. Paris: Hachette Littératures: 430-481.

Itard, Jean Marc Gaspard 1894. Rapports et mémoires sur le sauvage de l'Aveyron, l'Idiotie et la surdi-mutité. Paris: Progrès Médical.

Jainchill, Andrew 2008. Reimagining Politics after the Terror. Ithaca, NY u. a.: Cornell University Press.

Jamin, Jean 1982. Le syndrome chinois des idéologues ou les débuts de la sociolinguistique. Histoire Épistémologie Langage (4/1): 83-92.

Kaufmann, Doris 1995. Aufklärung, bürgerliche Selbsterfahrung und die „Erfindung“ der Psychiatrie in Deutschland, 1770-1850. Göttingen: Vandenhoeck \& Ruprecht.

Lane, Harlan 1999 [1992]. The Mask of Benevolence. Disabling the Deaf Community. San Diego: DawnSignPress.

Lanthenas, François Xavier 1792. De l'influence de la liberté sur la santé, la morale et le bonheur. Chronique du mois ou les cahiers patriotiques (Juni).

Lerner, Paul 1997. Healing and the Mind. In: Roy Porter (Hg.). Medicine. A History of Healing. Ancient Traditions to Modern Practices. London: Ivy Press: 144-167.

Leterrier, Sophie-Anne 1995. L'institution des sciences morales. L'Académie des sciences morales et politiques 1795-1850. Paris: L'Harmattan.

Lezay-Marnésia, Adrian 1797. Des causes de la Révolution et de ses résultats. Paris: Imprimerie du Journal d'économie publique.

Messling, Markus 2013. Text und Bestimmung. Determinismus und Rassenlogik in der Philologie. In: Markus Messling und Ottmar Ette (Hg.). Wort-Stamm-Macht. Rassismus und Determinismus in der Philologie (18./19. Jh.). München: Fink: 31-53.

Osterhammel, Jürgen 1989. China und die Weltgesellschaft. Vom 18. Jahrhundert bis in unsere Zeit. München: Beck.

Ozouf, Mona 1988a. La fête révolutionnaire. 1789-1799. Paris: Gallimard.

Ozouf, Mona 1988b. La Révolution française et l'idée de l'homme nouveau. In: Colin Lucas (Hg.). The Poltical Culture of the French Revolution. Oxford u.a.: Pergamon Press: 213-232.

Paradis, André 1993. De Condillac à Pinel ou les fondemens philosophiques du traitement moral. Philosophiques (1): 69-112.

Petit, Marc Antoine 1806 [1797]. Sur l'influence de la Révolution française sur la santé publique. In: ders. Essai sur la médecine du cœur. Lyon: Chez Garnier/Chez Reymann: $116-157$.

Peuple de Tours 1794. Adresse an die Convention nationale. Moniteur (316, 16 Thermiddor an II/03.08.1794): 375.

Pigeaud, Jackie 1980. Le rôle des passions dans la pensée médicale de Pinel à Moreau de Tours. History and Philosophy of the Life Sciences (2/1): 123-140.

Pinel, Philippe 1798. Recherches et observations sur le traitement moral des aliénés. s.l.

Quartararo, Anne T. 2008. Deaf Identity and Social Images in Nineteenth-Century France. Washington: Gallaudet University Press. 
Quinlan, Sean M. 2004. Physical and Moral Regeneration after the Terror. Medical Culture, Sensibility and Family Politics in France, 1794-1804. Social History (29/2): 139-164.

Roberts, J.A.G. 1997. L'image de la Chine dans l'Encyclopédie. Recherches sur Diderot et sur l'Encyclopédie (22): 87-108.

Rosen, George 1959. Social Stress and Mental Disease from the Eighteenth Century to the Present. Some Origins of Social Psychiatry. The Millibank Memorial Fund Quarterly (37/1): 5-32.

Rosenfeld, Sophia 1997. Deaf Men on Trial. Language and Deviancy in Late EighteenthCentury France. Eighteenth-Century Life (21/2): 157-175.

Rosenfeld, Sophia 2001. A Revolution in Language. The Problems of Signs in Late EighteenthCentury France. Stanford: Stanford University Press.

Sauveur, Toussaint-Dieudonné 1809. Rapport sur les travaux des membres correspondans \& sur les mémoires envoyés au concours. In: Société libre des sciences physiques \& médicales de Liege (Hg.). De l'influence des passions sur la production des maladies. Lüttich: Chez J. Desoer: 29-37.

Schacht, Johannes Oosterdyk 1733. Dissertatio medica inauguralis de terrore ejusque effectis in corpus humanum [...]. Utrecht: Alexander van Megen.

Scheidemantel, Friedrich Christian Gottlieb 1787. Die Leidenschaften als Heilmittel betrachtet. Hildburghausen: Johann Gottfried Hanisch.

Sicard, Roch Ambroise 1835 [1797]. Relation adressée par M. Abbé Sicard, Instituteur des sourds et muets, à un de ses amis, sur les dangers qu'il a courus les 2 et 3 septembre 1792. In: P.-J.-B. Buchez und P.-C. Roux (Hg.). Histoire parlamentaire de la Révolution Française. Bd. 18. Paris: Paulin: 72-103.

Sicard, Roch-Ambroise Cocurron de 1799. Cours d'instruction d'un sourd-muet de naissance, pour servir a l'éducation des sourds-muets. Paris: Le Cere.

Sieyès, Emmanuel Joseph 2010 [1789]. Was ist der Dritte Stand? In: ders., Was ist der Dritte Stand? Ausgewählte Schriften. Hg. v. Oliver W. Lembcke und Florian Weber, übersetzt von Andreas Fliedner, Oliver Lembcke und Florian Weber. Berlin: Akademie: 111-193.

Sieyès, Emmanuel Joseph 1789. Qu'est-ce que le tiers-état? s.l.

Staum, Martin S. 1996. Minerva's Message. Stabilizing the French Revolution. Montreal u. a.: McGill-Queen's University Press.

Steinberg, Ronen 2015. Trauma and the Effects of Mass Violence in Revolutionary France. Historical Reflections (41/3): 28-46.

Swain, Gladys 1997. Le sujet de la folie. Naissance de la psychiatrie. Paris: Calmann-Lévy.

Tableau des opérations de l'Assemblée nationale d'après le Journal de Paris et auquel on a ajouté tous les Articles de cette feuille, qui peuvent intéresser les arts $\mathcal{E}$ les sciences 1789-1792. Lausanne: Chez Hignou \& Comp.

Talleyrand-Périgord, Charles-Maurice de 1791. Rapport sur l'instruction publique fait au nom du Comité de constitution a l'assemblée nationale. Paris: Baudouin/Du Pont.

Tallien, Jeam Lambert 1794. Rede vor dem Comité du salut public der Convention nationale. Moniteur (343, 13 Fructidor an II/30.08.1794): 612-615.

Vecchio, Sebastiano 1986. Langue nationale et grammaire pendant la Révolution. La France et l'Italie. In: Winfried Busse und Jürgen Trabant (Hg.). Les Idéologues. Sémiotique, théories et politiques linguistiques pendant la Révolution française. Amsterdam/ Philadelphia: 373-395.

Voisin, Félix 1826. Des causes morales et physiques des maladies mentales et de quelques autres affections nerveuses. Paris: chez J.-B. Baillière.

Wagner, Peter 1998. Certainty and Order, Liberty and Contingency. The Birth of Social Science as Empirical Political Philosophy. In: Johan Heilbron, Lars Magnusson und Björn Wittrock (Hg.). The Rise of the Social Sciences and the Formation of Modernity. Conceptual Change in Context, 1750-1850. Dordrecht: Springer: 241-263.

Wahnich, Sophie 2009. Les émotions, la Révolution française et le présent. Exercices pratiques de conscience historique. Paris: CNRS Éd.

Weiner, Dora 2008. The Madman in the Light of Reason. Enlightenment Psychiatry. Part II: Alienists, Treatises, and the Psychologic Approach in the Era of Pinel. In Edwin Wallace und John Gach (Hg.). History of Psychiatry and Medical Psychology. New York: Springer: 281-304. 
Wokler, Robert 2000. From the Moral and Political Sciences to the Sciences of Society by Way of the French Revolution. Annual Review of Law and Ethics (8): 33-45.

Laurens Schlicht

Institut für Kulturwissenschaft

Humboldt-Universität zu Berlin

Unter den Linden 6

10099 Berlin

Deutschland

laurens.schlicht@hu-berlin.de 